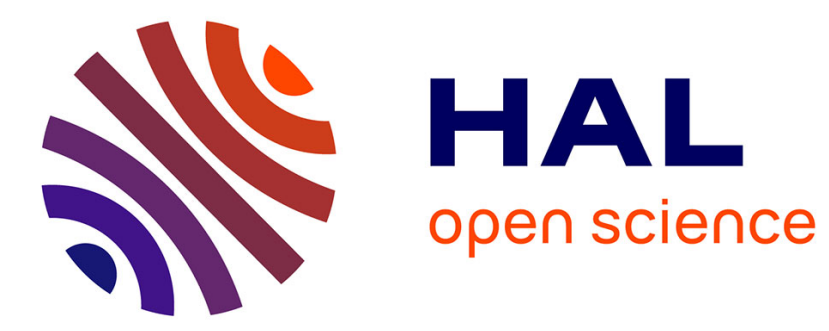

\title{
Annular acoustic black holes to reduce sound radiation from cylindrical shells
}

Jie Deng, Oriol Guasch, Laurent Maxit, Ling Zheng

\section{To cite this version:}

Jie Deng, Oriol Guasch, Laurent Maxit, Ling Zheng. Annular acoustic black holes to reduce sound radiation from cylindrical shells. Mechanical Systems and Signal Processing, 2021, 158, pp.107722. 10.1016/j.ymssp.2021.107722 . hal-03176274

\section{HAL Id: hal-03176274 \\ https://hal.science/hal-03176274}

Submitted on 15 Nov 2021

HAL is a multi-disciplinary open access archive for the deposit and dissemination of scientific research documents, whether they are published or not. The documents may come from teaching and research institutions in France or abroad, or from public or private research centers.
L'archive ouverte pluridisciplinaire HAL, est destinée au dépôt et à la diffusion de documents scientifiques de niveau recherche, publiés ou non, émanant des établissements d'enseignement et de recherche français ou étrangers, des laboratoires publics ou privés. 


\title{
Annular acoustic black holes to reduce sound radiation from cylindrical shells
}

\author{
Jie Deng ${ }^{\mathrm{a}, \mathrm{b}}$, Oriol Guasch ${ }^{\mathrm{b}, *}$, Laurent Maxit ${ }^{\mathrm{c}}$, Ling Zheng ${ }^{\mathrm{a}}$ \\ ${ }^{a}$ State Key Laboratory of Mechanical Transmission, College of Automotive Engineering \\ Chongqing University, Chongqing 400044, PR China \\ ${ }^{b}$ GTM - Grup de Recerca en Tecnologies Mèdia, La Salle, Universitat Ramon Llull \\ C/ Quatre Camins 30, 08022 Barcelona, Catalonia (Spain) \\ ${ }^{c}$ INSA Lyon, Laboratoire Vibrations Acoustique (LVA) \\ 25 bis, av. Jean Capelle, F-69621 Villeurbanne Cedex, France
}

\begin{abstract}
Annular acoustic black holes (ABHs) have been recently proposed as a potential means of reducing vibrations of cylindrical shells. The latter are very common structures in the naval, aeronautic and industrial sectors so widening $\mathrm{ABH}$ applications from flat plates to curved structures seems worth exploring. This work focuses on the benefits of embedding annular ABH indentations on cylindrical shells to reduce outward sound radiation. The goal of the paper is to propose a semi-analytical method to determine the acoustic power, radiation efficiency, source location and far-field acoustic pressure of $\mathrm{ABH}$ shells and compare them with those of uniform thickness shells. The vibration field of the ABH and uniform cylindrical shells is computed by means of the Gaussian expansion method (GEM) within the Rayleigh-Ritz approach. Then, the radiated pressure is obtained by solving the Helmholtz equation in cylindrical coordinates using the Green's function method. The surface of the cylinder is discretized into small finite size radiators and an impedance matrix is used to obtain the acoustic surface pressure in terms of the shell radial velocity. To determine those regions of the cylinder responsible for the far field radiated sound, use is made of supersonic sound intensity (SSI). A method is proposed to calculate the SSI in the spatial domain for cylindrical shell structures to make direct use of the previously computed surface pressure and velocity distributions. The whole methodology is validated against finite element method (FEM) simulations and after that, results are presented for an acoustically thick shell. The roles played by the critical and ring frequencies are reported and the spectra of the acoustic power, radiation efficiency and far field acoustic pressure get analysed. It is shown that the annular $\mathrm{ABH}$ can become very effective once the cylinder flexural motion dominates over the circumferential one. The slow down of bending waves inside the ABH makes structural supersonic waves (in relation to sound speed) become subsonic at some point, which clearly diminishes the shell radiation efficiency. Overall, it is described why embedding an annular $\mathrm{ABH}$ on a cylindrical shell can strongly help reducing the radiated sound to the far-field.
\end{abstract}

Keywords: Annular acoustic black holes, Gaussian expansion method, Cylindrical shells, Sound radiation, Supersonic intensity

\section{Introduction}

While embedding acoustic black holes (ABHs) in structures has proved to be a light-weight, efficient method for reducing vibrations, as well as for wave manipulation and energy harvesting (see e.g., the very recent and complete review in [1]), not many works have yet considered the potential of ABHs to diminish structural radiated sound. One of the first works in this line was that in [2], where a combination of finite

\footnotetext{
*Corresponding Author: oriol.guasch@salle.url.edu
} 
element (FEM) and boundary element (BEM) methods were used to investigate the reduction in sound power level and radiated sound when embedding an array of ABHs on a flat plate. By transforming to the wavenumber domain, it was lately shown in [3] that when supersonic flexural waves enter an $\mathrm{ABH}$ and get slowed down, they eventually become subsonic which strongly diminishes the radiation efficiency of $\mathrm{ABH}$ plates as compared to plates of uniform thickness. More recently, a semi-analytical method was proposed in [4] in which the vibration field of the $\mathrm{ABH}$ plate was determined using wavelets as basis functions for the Rayleigh-Ritz method, and then input in Rayleigh's integral to compute the radiated sound. Supersonic intensity (SSI) [5, 6] was employed to find the location on the ABH surface of sources responsible for farfield sound radiation. The same authors resorted to topological optimization in [7] to properly distribute damping material within the $\mathrm{ABH}$ to minimize the radiated sound. Likewise, the benefits of embedding periodic tunnelled double-leaf $\mathrm{ABHs}$ on flat plates to decrease sound emission was analysed in [8]. On the other hand, the vibroacoustics of somewhat more complex systems including ABH indentations has begun to be explored. For instance, in [9] the case of an $\mathrm{ABH}$ plate coupled to a cavity was addressed, and the transmission loss (TL) of ABH plates was numerically and experimentally investigated in [10, 11]. Moreover, the $\mathrm{TL}$ of $\mathrm{ABH}$ plates in the mid-high frequency range has been recently explored by means of statistical modal energy distribution analysis (SmEdA) [12]. Several of the theoretical conclusions in the preceding articles have been supported by experimental evidence, see e.g., [8, 10, 13-15].

All the above cited works deal with $\mathrm{ABH}$ indentations on flat plates. In fact, with the exception of the spiral ABH design in [16, 17], which is an interesting concept but of difficult practical implementation, most current proposals to exploit the $\mathrm{ABH}$ effect consider different types of indentations on plates. The latter all share a common decreasing power-law thickness profile but adapted to different geometries. Without any doubt, the most familiar one consists of 2D circular indentations (see e.g., [18-22]) but other geometries like rectangular [23], double-layer compound [8, 24] or ring-shaped ABHs [25] have been proposed as alternatives, or to address particular problems. Indeed, if one thinks of potential practical applications of ABHs, it is worth considering even further designs. For instance, in the naval, aeronautic and industrial sectors, cylindrical shells are very common so embedding $\mathrm{ABHs}$ on curved structures deserves exploration. A first work in that direction was presented in [26], where several ABH indentations were placed on a circular beam. That resulted in typical phenomena of periodic systems, like the formation of frequency bandgaps reported for infinite periodic $\mathrm{ABH}$ beams in [27]. Another design has been recently proposed in [28-30] consisting of an annular ABH which aims at reducing vibrations on cylindrical shells. A FEM investigation was carried out in $[28,29]$ to check its performance when submitted to simply supported periodic boundary conditions, and a semi-analytical approach was suggested in [30] to carry out detailed parametric analyses of the annular ABH cylinder vibration field.

The main goal and contribution of this paper is to propose a semi-analytical approach to determine the effects of annular ABHs on sound radiated by vibrating cylindrical shells. In particular, we want to see how the cylinder sound power level and radiation efficiency get modified because of the ABH. Also, we would like to locate the sources of far-field radiated sound on the ABH cylindrical shell surface and compute the acoustic pressure at any distance from the shell. Nonetheless, before outlining the procedure that will be followed to achieve such objectives let us briefly recall a few basic facts of the vibroacoustics of cylindrical shells. Because of its industrial interest many works exist on the topic. Analytical methods initially contemplated the ideal case of infinite length cylinders (see e.g., [31]), though in most practical cases the finite length of the structure and boundary conditions play an essential role in the vibroacoustics of cylindrical shells [32]. In a nutshell, the latter can be categorized as being acoustically thin, when their critical frequency $f_{c}$ is bigger than their ring frequency $f_{R}$, (i.e., $f_{R} / f_{c}<1$ ) or as acoustically thick if $f_{R} / f_{c}>1$. These two frequencies play an important role in cylinder sound radiation. For frequencies larger than the critical one, flexural wave motion becomes supersonic (with respect to sound wave speed) and radiates sound very efficiently [33], while the opposite occurs for frequencies smaller than $f_{c}$. On the other hand, the ring frequency determines the threshold beyond which the cylindrical shell curvature begins losing importance and its vibration starts resembling that of a flat plate (see e.g., [34]). In general, acoustic thin shells are easier to characterize because they all follow the same vibroacoustic trends but unfortunately most cylindrical shells of practical interest are acoustically thick [32]. Note that being acoustically thick does not mean that thin shell theory cannot be applied; the two concepts are distinct. Thin shell theory is valid whenever the 
thickness of the cylindrical shell is much smaller than its radius. Dealing with acoustic thick shells to which thin shell theory applies is totally standard and the examples in this work address such situation. As said however, the vibroacoustics of acoustic thick shells are complex and can depend on many factors including the boundary conditions, shell and stiffeners geometry and types of excitation [32]. Concerning the latter, several of them have been considered in literature: from mechanical point or distributed forces, acoustic incident waves or turbulent boundary layers. The reader is referred e.g., to [35, 36] and references therein for specific bibliography on the topic. Being this the first work on sound radiated by a cylindrical shell with an embedded annular ABH, only mechanical point excitation will be considered.

To characterize the sound radiated by the annular ABH cylindrical shell we first need to compute its vibration field when submitted to the external point excitation. This will be done using Gaussian basis functions in the framework of the Rayleigh-Ritz method and the procedure will be referred to as the GEM [37, 38]. The GEM was initially developed for straight beams [37] and plates [38] but adapted to circular beams in [26] and to cylindrical shells in [30]. The GEM gets its inspiration from wavelet approaches for ABH beams and plates (see e.g., [39-41]) and has the advantage of easily dealing with periodic boundary conditions, as those encountered in the circumferential direction of a cylinder. Once the surface velocity of the ABH shell is computed, the next step is to obtain the surface acoustic pressure distribution to determine the shell's acoustic power. This can be done by solving the Helmholtz equation with the Green's function in cylindrical coordinates and defining and impedance matrix that relates the shell surface velocity with the acoustic pressure [42, 43]. The ABH shell acoustic power and radiation efficiency can be obtained from the surface velocity and pressure distribution. After that, we shall focus in locating the sources of far-field sound radiation on the $\mathrm{ABH}$ cylindrical shell. To that purpose one can resort to supersonic intensity (SSI), which relies on transforming the pressure and velocity fields to the wavenumber domain and eliminate the effects of subsonic propagating flexural waves to build the intensity, which are mostly evanescent and do not contribute to the far-field $[5,6,44]$. As we are working in the spatial domain and not in the wavenumber one, it would be interesting to formulate SSI in the former. This was actually done for flat plates in [45] and applied in [4] to locate sound sources in a plate with embedded 2D circular ABHs. In this work we will develop a methodology to compute the SSI for cylindrical shells in the spatial domain that can serve for the annular ABH cylindrical shell. It is to be noted that alternatives to improve the outcomes of SSI have been proposed in literature such as non-negative intensity [46-48], though we will limit to SSI in this work. Finally, the acoustic pressure at any point in the far field will be computed by means of the solution to the Helmholtz equation mentioned above. Comparisons of all computed quantities for the ABH annular shell will be compared with those of a uniform shell to illustrate the benefits of the former.

The remaining of this paper is organized as follows. In Section 2 we first state the radiation problem we want to solve and then present a reminder of the GEM developments in [30] to characterize the vibration field of the annular ABH cylindrical shell. Next, the sound radiation model together with the acoustic impedance matrix to compute the acoustic power and radiation efficiency are introduced. This is followed by the proposed strategy to deal with SSI in the spatial domain for cylindrical geometries. The complete semi-analytical approach for sound radiation is validated against FEM simulations in Section 3 . Then we proceed to analyse the vibroacoustic performance of the annular $\mathrm{ABH}$ in Section 4. The results for mean surface radial velocity, acoustic power, sound power level, radiation efficiency, intensity, supersonic intensity and surface and far-field acoustic pressure are reported and compared for $\mathrm{ABH}$ and uniform cylindrical shells. Conclusions are finally drawn in Section 5.

\section{Sound radiation model for ABH cylindrical shells}

\subsection{Statement of the problem}

To begin with, let us describe the radiation problem addressed in this paper in some detail. We consider a cylindrical shell submitted to an external radial point force excitation (see Fig. 1). As a result, the shell vibrates and emits sound waves that propagate outwards. For simplicity, the shell radiating in air is baffled between two semi-infinite rigid cylinders (see Fig. 1a), so no interaction occurs at edges between the inner and outer shell acoustic fields. In this work, we are only interested in the second one, i.e., on exterior acoustic radiation. 

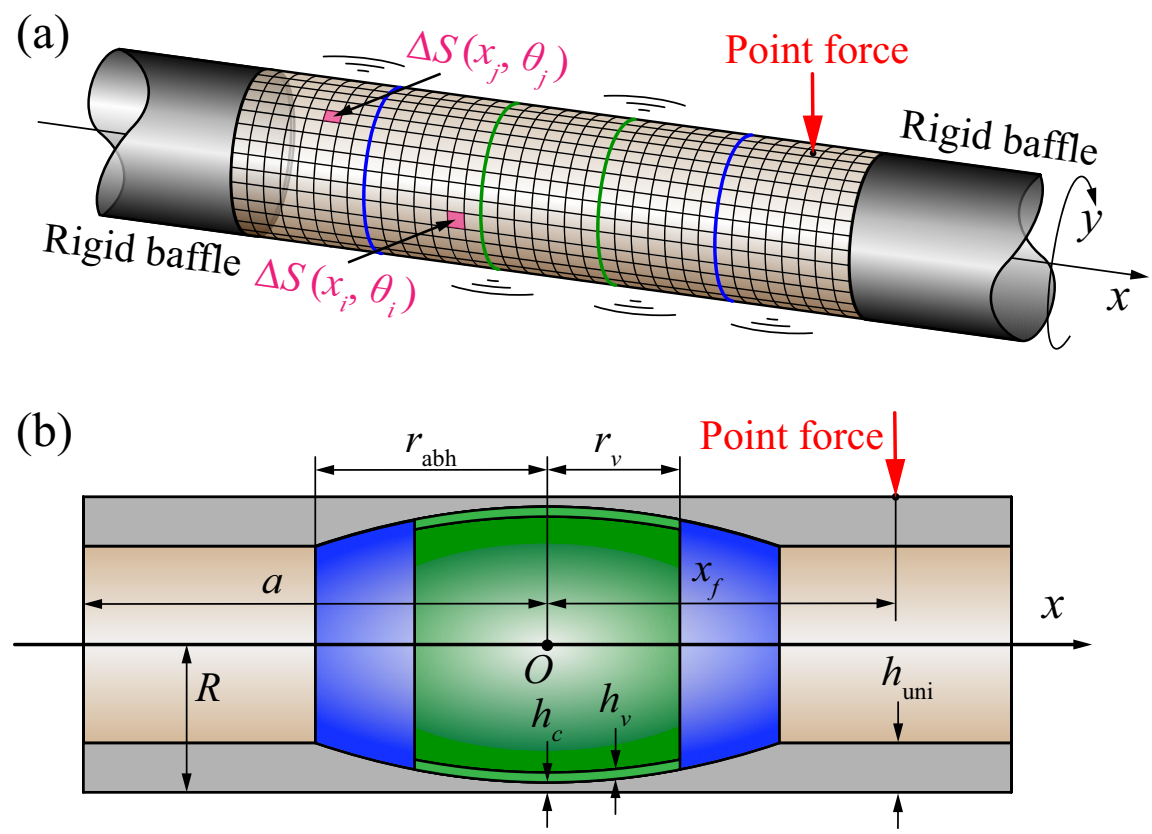

Figure 1: a) Baffled cylinder with internal annular ABH. Division into elementary patches $\Delta S$ to compute sound radiation. b) Section of the cylindrical shell with the annular ABH indentation.

To reduce the sound radiated by the cylindrical shell we consider embedding an annular ABH at its interior comprising the space between the two blue circles in Fig. 1a. Flexural waves entering the ABH are expected to slow down and increase their amplitude while approaching the ABH central region. There, energy becomes dissipated by means of a damping layer that covers the area delimited by the green circles in Fig. 1a. Our goal is to investigate how the reduction of shell vibrations induced by the ABH effect influence the shell sound power level, its radiation efficiency and the emission of acoustic waves to the far field.

The cylindrical shell has external radius $R$, thickness $h_{\text {uni }}$ and extends between $-a<x<a$. The axial direction of the cylinder is denoted by $x$ and the circumferential one by $y$, with $y=\theta R$ and $\theta$ being the angle in cylindrical coordinates. The junction between the shell and the semi-infinite rigid baffles is chosen to be elastic and can accommodate various types of boundary conditions by tuning its parameters. The geometric details of the annular $\mathrm{ABH}$ are depicted in Fig. 1b. It has half length $r_{\mathrm{abh}}$ and a power-law varying thickness $h(x)=\varepsilon|x|^{m}+h_{c}$, where $m$ and $h_{c}$ respectively designate the ABH order and the central truncation thickness. $\varepsilon=\left(h_{\mathrm{uni}}-h_{c}\right) r_{\mathrm{abh}}^{-m}$ stands for the smoothness parameter, which characterizes the slope of the $\mathrm{ABH}$ profile. In what concerns the damping layer, it has half length $r_{v}$ and a uniform thickness $h_{v}$. The external excitation force is located at point $\boldsymbol{x}_{f}=\left(x_{f}, y_{f}\right)$.

\subsection{Vibration field of the annular ABH cylindrical shell}

To determine the sound radiated by an ABH cylindrical shell when submitted to an external excitation, we first need to know its surface velocity distribution. As the shell radiates into air, it is well-known that the coupling between the shell and the acoustic domain is weak so one can estimate the shell vibration neglecting air's influence. To obtain the shell's surface velocity we rely on the Gaussian expansion method (GEM). The GEM applied to ABH cylindrical shells was described in the authors' previous work [30] and only a summary will be presented hereafter for the article completeness. The reader is referred to [30] for further details.

Unlike flat plates, where transverse vibration is clearly dominant in terms of sound radiation and inplane motion can often be discarded, vibrations in the three directions must be accounted for in the case of cylinders, as these are usually strongly coupled. This means that we have to deal with the axial displacement $u$, the circumferential displacement $v$, and the radial displacement $w$. 
Each of the three displacements $u, v, w$ can be decomposed in a series of basis functions. Let us respectively symbolize the basis functions in the three directions as $\psi_{i}(x, y), \xi_{i}(x, y), \varphi_{i}(x, y)$, with a priori unknown weight coefficients $a_{i}(t), b_{i}(t), c_{i}(t)$. It follows,

$$
\begin{aligned}
u(x, y, t) & =\sum_{i} a_{i}(t) \psi_{i}(x, y)=\mathbf{a}^{\top} \boldsymbol{\psi}=\boldsymbol{\psi}^{\top} \mathbf{a}, \\
v(x, y, t) & =\sum_{i} b_{i}(t) \xi_{i}(x, y)=\mathbf{b}^{\top} \boldsymbol{\xi}=\boldsymbol{\xi}^{\top} \mathbf{b}, \\
w(x, y, t) & =\sum_{i} c_{i}(t) \varphi_{i}(x, y)=\mathbf{c}^{\top} \boldsymbol{\varphi}=\boldsymbol{\varphi}^{\top} \mathbf{c},
\end{aligned}
$$

with

$$
\mathbf{a}=\hat{\mathbf{A}} \exp (\mathrm{j} \omega t), \mathbf{b}=\hat{\mathbf{B}} \exp (\mathrm{j} \omega t), \mathbf{c}=\hat{\mathbf{C}} \exp (\mathrm{j} \omega t) .
$$

as we are assuming harmonic time dependence. In Eqs. (1)-(3), $\boldsymbol{\psi}, \boldsymbol{\xi}$ and $\boldsymbol{\varphi}$ are column vectors with respective entries $\psi_{i}(x, y), \xi_{i}(x, y)$ and $\varphi_{i}(x, y)$. Introducing Kronecker's product, vectors $\boldsymbol{\psi}, \boldsymbol{\xi}$ and $\boldsymbol{\varphi}$ can be factorized as

$$
\begin{gathered}
\boldsymbol{\psi}(x, y)=\boldsymbol{\alpha}^{\psi}(x) \otimes \boldsymbol{\beta}^{\psi}(y), \\
\boldsymbol{\xi}(x, y)=\boldsymbol{\alpha}^{\xi}(x) \otimes \boldsymbol{\beta}^{\xi}(y), \\
\boldsymbol{\varphi}(x, y)=\boldsymbol{\alpha}^{\varphi}(x) \otimes \boldsymbol{\beta}^{\varphi}(y),
\end{gathered}
$$

where $\boldsymbol{\alpha}^{(i)}(i=\psi, \xi, \varphi)$ are column vectors containing basis functions that only depend on the $x$ direction and $\boldsymbol{\beta}^{(i)}(i=\psi, \xi, \varphi)$ are analogous column vectors only depending on the $y$ direction. The entries of $\boldsymbol{\alpha}$ and $\boldsymbol{\beta}$ are given by

$$
\begin{aligned}
\alpha_{i}(x) & =2^{s_{x} / 2} \exp \left[-\left(2^{s_{x}} x-q_{x_{i}}\right)^{2} / 2\right], \\
\beta_{i}(y) & =2^{s_{y} / 2} \exp \left[-\left(2^{s_{y}} y-q_{y_{i}}\right)^{2} / 2\right],
\end{aligned}
$$

and are obtained by translation and dilation of a general Gaussian function $g(z)=\exp \left(-z^{2} / 2\right)($ see $[37,38]$ for details on how to build the Gaussian basis). However, as quoted in [30], Eq. (9) cannot be directly used to decompose the vibration in the $y$ direction because periodicity has to be imposed in that direction. That is, the following conditions must be satisfied,

$$
\begin{aligned}
u(x, 0) & =u(x, 2 \pi R), v(x, 0)=v(x, 2 \pi R), w(x, 0)=w(x, 2 \pi R), \\
\frac{\partial u}{\partial y}(x, 0) & =\frac{\partial u}{\partial y}(x, 2 \pi R), \frac{\partial v}{\partial y}(x, 0)=\frac{\partial v}{\partial y}(x, 2 \pi R), \frac{\partial w}{\partial y}(x, 0)=\frac{\partial w}{\partial y}(x, 2 \pi R), \\
\frac{\partial^{2} u}{\partial y^{2}}(x, 0) & =\frac{\partial^{2} u}{\partial y^{2}}(x, 2 \pi R), \frac{\partial^{2} v}{\partial y^{2}}(x, 0)=\frac{\partial^{2} v}{\partial y^{2}}(x, 2 \pi R), \frac{\partial^{2} w}{\partial y^{2}}(x, 0)=\frac{\partial^{2} w}{\partial y^{2}}(x, 2 \pi R) .
\end{aligned}
$$

As shown in [30], after some manipulations the Gaussian basis in Eqs. (8)-(9) can be modified to satisfy Eqs. (10)-(12).

To determine the displacement field $(u, v, w)$ from the Rayleigh-Ritz method, we next need to find expressions for the kinetic and potential energy of the ABH cylindrical shell. The kinetic energy is given by,

$$
K_{\text {shell }}=\frac{1}{2} \int_{0}^{2 \pi R} \int_{-a}^{a} \rho h(x, y)\left[\left(\frac{\partial u}{\partial t}\right)^{2}+\left(\frac{\partial v}{\partial t}\right)^{2}+\left(\frac{\partial w}{\partial t}\right)^{2}\right] \mathrm{d} x \mathrm{~d} y
$$

where $\rho$ and $h(x, y)$ represent the shell density and local thickness, respectively. 
The potential energy of the shell reads (see e.g. [49]),

$$
\begin{aligned}
U_{\text {shell }}=\frac{1}{2} \int_{0}^{2 \pi R} \int_{-a}^{a} D(x, y) & {\left[\left(\frac{\partial^{2} w}{\partial x^{2}}\right)^{2}+\left(\frac{\partial^{2} w}{\partial y^{2}}-\frac{1}{R} \frac{\partial v}{\partial y}\right)^{2}\right.} \\
& \left.+2 \nu \frac{\partial^{2} w}{\partial x^{2}}\left(\frac{\partial^{2} w}{\partial y^{2}}-\frac{1}{R} \frac{\partial v}{\partial y}\right)+\frac{1-\nu}{2}\left(2 \frac{\partial^{2} w}{\partial x \partial y}-\frac{2}{R} \frac{\partial v}{\partial x}\right)^{2}\right] \mathrm{d} x \mathrm{~d} y \\
+\frac{1}{2} \int_{0}^{2 \pi R} \int_{-a}^{a} G(x, y)\left\{\left(\frac{\partial u}{\partial x}\right)^{2}+\left(\frac{\partial v}{\partial y}+\frac{w}{R}\right)^{2}\right. & \left.+\nu\left[\frac{\partial u}{\partial x}\left(\frac{\partial v}{\partial y}+\frac{w}{R}\right)+\left(\frac{\partial v}{\partial y}+\frac{w}{R}\right) \frac{\partial u}{\partial x}\right]+\frac{1-\nu}{2}\left(\frac{\partial v}{\partial x}+\frac{\partial u}{\partial y}\right)^{2}\right\} \mathrm{d} x \mathrm{~d} y
\end{aligned}
$$

where $D(x, y)=\frac{E^{\star} h^{3}(x, y)}{12\left(1-\nu^{2}\right)}$ is the bending stiffness and $G(x, y)=\frac{E^{\star} h(x, y)}{1-\nu^{2}}$ the extensional one. $E^{\star}=E(1+\mathrm{i} \eta)$ stands for the complex Young modulus that includes the material loss factor $\eta$.

A large variety of boundary conditions can be simulated by assigning different values to translational springs with stiffness values $k_{i}^{j}, i=1,2, j=u, v, w$, placed at the edges of the cylinder, as well as to rotational springs with parameters $p_{i}^{w}, i=1,2$. Their contribution to the potential energy has to be considered and turns to be,

$$
\begin{aligned}
U_{\text {edge }}= & \frac{1}{2} \int_{0}^{2 \pi R}\left\{k_{1}^{w} u^{2}(-a, y)+k_{1}^{v} v^{2}(-a, y)+k_{1}^{w} w^{2}(-a, y)+p_{1}^{w}\left[\frac{\partial w}{\partial x}(-a, y)\right]^{2}\right\} \mathrm{d} y \\
& +\frac{1}{2} \int_{0}^{2 \pi R}\left\{k_{2}^{u} u^{2}(a, y)+k_{2}^{v} v^{2}(a, y)+k_{2}^{w} w^{2}(a, y)+p_{2}^{w}\left[\frac{\partial w}{\partial x}(a, y)\right]^{2}\right\} \mathrm{d} y .
\end{aligned}
$$

Finally, the external work resulting from the point force at $\boldsymbol{x}_{f}=\left(x_{f}, y_{f}\right)$ is expressed as

$$
W=f(t) w\left(x_{f}, y_{f}, t\right) .
$$

Eqs. (13)-(16) are valid for a cylindrical shell with any varying thickness profile and with arbitrary boundary conditions. The influence of damping layers can be incorporated in the model following the fullycoupled method [39]. Analogous expressions to Eqs. (13)-(14) are used to determine the viscous damping layer kinetic, $K_{\text {vis }}$, and potential $U_{\text {vis }}$, energies.

The complete Lagrangian of the system is built as

$$
\mathscr{L}=K-U+W=\left(K_{\text {shell }}+K_{\text {vis }}\right)-\left(U_{\text {shell }}+U_{\text {vis }}+U_{\text {edge }}\right)+W=\frac{1}{2} \dot{\mathbf{q}}^{\top} \mathbf{M} \dot{\mathbf{q}}-\frac{1}{2} \mathbf{q}^{\top} \mathbf{K q}+\mathbf{q}^{\top} \mathbf{f}
$$

where in the last equality we have inserted the expansions Eqs. (1)-(3) into Eqs. (13)-(16) and their equivalent for the damping layer. The vector

$$
\mathbf{q}=\left[\hat{\mathbf{A}}^{\top}, \hat{\mathbf{B}}^{\top}, \hat{\mathbf{C}}^{\top}\right]^{\top} \exp (\mathrm{j} \omega t) \equiv \hat{\mathbf{Q}} \exp (-\mathrm{j} \omega t),
$$

in Eq. (17) collects the system unknowns of Eq. (4), while $\mathbf{M}$ represents the mass matrix, $\mathbf{K}$ the stiffness one and $\mathbf{f}=\hat{\mathbf{F}} \exp (-\mathrm{j} \omega t)$ the force vector. Their detailed expressions are provided in [30] as well as an explanation on how to embed the annular ABH on them according to the matrix replacing strategy in [38]. The equations of motion for the $\mathrm{ABH}$ cylindrical shell are obtained from the Euler-Lagrange equations $\partial_{t}\left(\partial_{\dot{q}} \mathscr{L}\right)-\partial_{\boldsymbol{q}} \mathscr{L}=0$, which yield, in the frequency domain,

$$
\left(-\omega^{2} \mathbf{M}+\mathbf{K}\right) \hat{\mathbf{Q}}=\hat{\mathbf{F}} .
$$

The solution to Eq. (19) provides the displacement field of the ABH cylindrical shell at a given angular frequency $\omega$. 


\subsection{Sound radiation and supersonic intensity}

\subsubsection{Acoustic pressure, sound power and radiation efficiency}

Once the radial displacement $w(x, y)$ is computed, the normal velocity to the cylinder surface can be simply obtained as $v_{w}(x, y)=-\mathrm{j} \omega w(x, y)$. For a baffled cylindrical shell, the radiated sound pressure at an external point $\left(x^{\prime}, y^{\prime}, r\right) \equiv\left(x^{\prime}, \theta^{\prime} R, r\right)$ due to the shell vibration can be calculated by means of the Helmholtz equation Green's function in cylindrical coordinates. This yields (see $[42,43])$,

$p\left(x^{\prime}, \theta^{\prime} R, r\right)=\frac{\mathrm{j} \rho_{0} \omega}{4 \pi^{2}} \int_{0}^{2 \pi} \int_{-a}^{a} v_{w}(x, \theta R) \sum_{n=0}^{+\infty} \epsilon_{n} \cos \left[n\left(\theta^{\prime}-\theta\right)\right] \int_{-\infty}^{+\infty} \frac{\exp \left[\mathrm{j} k_{x}\left(x^{\prime}-x\right)\right]}{k_{y} R} \frac{\mathrm{H}_{n}^{(1)}\left(\sqrt{k_{0}^{2}-k_{x}^{2}} r\right)}{\mathrm{H}_{n}^{(1) \prime}\left(\sqrt{k_{0}^{2}-k_{x}^{2}} R\right)} \mathrm{d} k_{x} \mathrm{~d} x R \mathrm{~d} \theta$,

where $\rho_{0}$ represents the air density, $k_{0}=\omega / c_{0}$ the total acoustic wavenumber ( $c_{0}$ is the speed of sound) and $n$ designates the circumferential number (besides, remember that $y=\theta R$ ). Likewise, $\mathrm{H}_{n}^{(1)}$ stands for the $n$-th Hankel function of the first kind while $\mathrm{H}_{n}^{(1) \prime}$ represents its first derivative with respect to the argument $k_{y} R$. The normalization coefficient $\epsilon_{n}$ in Eq. (20) has values,

$$
\epsilon_{n}= \begin{cases}1 & n=0 \\ 2 & n>0 .\end{cases}
$$

For the numerical estimation of Eq. (20), the cylindrical shell can be divided into $N$ equal elementary radiators of area $\Delta S$ (see Fig. 1). The normal velocity is assumed constant within each radiator and we can assemble all of them into an $N \times 1$ vector $\mathbf{v}_{w}$. Then, the sound pressure at the surface of each radiator (acoustic wall pressure) can be also expressed as an $N \times 1$ vector $\mathbf{p}$ such that,

$$
\mathbf{p}=\mathbf{Z v}_{w} .
$$

Here, $\mathbf{Z}$ represents the $N \times N$ acoustic impedance matrix with entries,

$$
Z_{i j}=\frac{\mathrm{j} \rho_{0} \omega \Delta \mathrm{S}}{2 \pi^{2}} \sum_{n=0}^{+\infty} \epsilon_{n} \cos \left[n\left(\theta_{i}-\theta_{j}\right)\right] \int_{0}^{+\infty} \frac{\cos \left[k_{x}\left(x_{i}-x_{j}\right)\right]}{\sqrt{k_{0}^{2}-k_{x}^{2}} R} \frac{\mathrm{H}_{n}^{(1)}\left(\sqrt{k_{0}^{2}-k_{x}^{2}} r\right)}{\mathrm{H}_{n}^{(1) \prime}\left(\sqrt{k_{0}^{2}-k_{x}^{2}} R\right)} \mathrm{d} k_{x} .
$$

On the other hand, the ABH cylinder sound power radiation can be computed as

$$
W_{\text {rad }}=\mathbf{v}_{w}^{\mathrm{H}} \mathbf{R} \mathbf{v}_{w},
$$

where the superscript $\mathrm{H}$ stands for the Hermitian transpose and $\mathbf{R}=\frac{\Delta S}{2} \operatorname{Re}\left(\left.\mathbf{Z}\right|_{r=R}\right)$ is the radiation resistance matrix, which is real, symmetric and positive-definite. Knowing $W_{\text {rad }}$, one can recover the radiation efficiency of the cylinder from its definition [31],

$$
\sigma=\frac{W_{\mathrm{rad}}}{\rho_{0} c_{0} N \Delta S\left\langle v_{w}^{2}\right\rangle_{S}},
$$

where $\left\langle v_{w}^{2}\right\rangle_{S}$ represents the mean square velocity (MSV) over the whole surface $S$ of the ABH cylindrical shell. The MSV can be directly computed from the normal velocities determined with the semi-analytical GEM approach of Section 2.2. $Z_{0}=\rho_{0} c_{0}$ in Eq. (25) is the air characteristic impedance, $c_{0}$ being the sound speed in air.

\subsubsection{Supersonic intensity in the spatial domain}

To identify the vibration regions on the cylinder surface that most effectively radiate sound to the far field, one can resort to supersonic intensity [5]. Hereafter, we will follow its formulation in the spatial domain, as done for a plate in [45], but adapting the strategy to the cylindrical problem at hand. As the 
procedure involves expressing the radial velocity and the acoustic wall pressure fields at the cylinder surface (i.e., $r=R$ ) in the wavenumber domain, we must first force them to be periodic in the circumferential direction $y=\theta R$. This can be done by convolving their expressions with a Dirac comb distribution (see e.g., [33] or [36] for recent applications in cylindrical shell vibroacoustics) so that,

$$
\begin{gathered}
\bar{v}_{w}(x, y) \equiv v_{w}(x, y) * \frac{1}{2 \pi R} \operatorname{III}\left(\frac{y}{2 \pi R}\right), \\
\bar{p}(x, y) \equiv p(x, y) * \frac{1}{2 \pi R} \operatorname{III}\left(\frac{y}{2 \pi R}\right),
\end{gathered}
$$

with

$$
\mathrm{III}(y / 2 \pi R)=2 \pi R \sum_{n=-\infty}^{+\infty} \delta(y-2 \pi R n) .
$$

As usual, $\delta$ stands for Dirac's delta and $*$ for the convolution operation.

To lighten the presentation, let us primarily focus on the velocity field, the developments for the pressure being totally analogous. The spatial Fourier transform of $\bar{v}_{w}(x, y)$ is given by

$$
\bar{V}_{w}\left(k_{x}, k_{y}\right)=\int_{-\infty}^{+\infty} \int_{-\infty}^{+\infty} \bar{v}_{w}(x, y) \mathrm{e}^{-\mathrm{j} k_{x} x} \mathrm{e}^{-\mathrm{j} k_{y} y} \mathrm{~d} x \mathrm{~d} y=V_{w}\left(k_{x}, k_{y}\right) \operatorname{III}\left(R k_{y}\right),
$$

where use has been made of Eq. (26) and the convolution theorem. $V_{w}\left(k_{x}, k_{y}\right)$ denotes the spatial Fourier transform of $v_{w}(x, y)$. The inverse transform of Eq. (29) reads,

$$
\bar{v}_{w}(x, y)=\frac{1}{(2 \pi)^{2}} \int_{-\infty}^{+\infty} \int_{-\infty}^{+\infty} V_{w}\left(k_{x}, k_{y}\right) \operatorname{III}\left(R k_{y}\right) \mathrm{e}^{\mathrm{j} k_{x} x} \mathrm{e}^{\mathrm{j} k_{y} y} \mathrm{~d} k_{x} \mathrm{~d} k_{y} .
$$

Supersonic intensity relies on the fact that, at least for unbounded structures, only waves propagating at supersonic speed on the structure (e.g., a cylinder in our case) are effective in radiating acoustic waves to the far field. The term supersonic makes reference to the speed of waves in the structure as compared to $c_{0}$, but obviously the generated acoustic waves propagate outwards at the speed of sound. This means that only structural waves with wavenumbers in the acoustic radiation disc $\bar{D}_{\text {rad }}=$ $\left\{\left(k_{x}, k_{y}\right) \in \mathbb{R} \mid k_{x}^{2}+k_{y}^{2} \leq k_{0}^{2}=\left(\omega / c_{0}\right)^{2}\right\}$ contribute to the far field, while those fulfilling $k_{x}^{2}+k_{y}^{2}>k_{0}^{2}$ are evanescent and can be discarded as potential sound sources. In the case of a plate, $k_{x}$ and $k_{y}$ in the radiation disc respectively refer to the standard wavenumber components in the $x$ and $y$ directions [6], while in the case of cylinders the radiation condition was only applied to the wavenumber in the axial direction, i.e., $k_{x}<k_{0}$, in [5]. In what follows, we will also consider the circumferential direction and proceed in the spatial domain as in [45]. It will be made apparent below that $k_{y}=n / R$, so that our radiation disc is given by $\bar{D}_{\text {rad }}=\left\{k_{x} \in \mathbb{R}, n \in \mathbb{N} \mid k_{x}^{2}+(n / R)^{2} \leq k_{0}^{2}=\left(\omega / c_{0}\right)^{2}\right\}$.

The supersonic velocity $\bar{v}_{w}^{(s)}(x, y)$ is defined by limiting the integral in Eq. (30) to values $\left(k_{x}, k_{y}\right) \in \bar{D}_{\text {rad }}$. That is,

$$
\bar{v}_{w}^{(s)}(x, y)=\frac{1}{(2 \pi)^{2}} \iint_{\bar{D}_{\mathrm{rad}}} V_{w}\left(k_{x}, k_{y}\right) \operatorname{III}\left(R k_{y}\right) \mathrm{e}^{\mathrm{j} k_{x} x} \mathrm{e}^{\mathrm{j} k_{y} y} \mathrm{~d} k_{x} \mathrm{~d} k_{y} .
$$

However, we can recover the infinite limits by introducing the Heaviside unit function,

$$
H\left(k_{x}, k_{y}\right)=\left\{\begin{array}{cl}
1 & \left(k_{x}, k_{y}\right) \in \bar{D}_{\mathrm{rad}} \\
0 & \left(k_{x}, k_{y}\right) \notin \bar{D}_{\mathrm{rad}}
\end{array}\right.
$$

to rewrite Eq. (31) as

$$
\bar{v}_{w}^{(s)}(x, y)=\frac{1}{(2 \pi)^{2}} \int_{-\infty}^{+\infty} \int_{-\infty}^{+\infty} V_{w}\left(k_{x}, k_{y}\right) \operatorname{III}\left(R k_{y}\right) H\left(k_{x}, k_{y}\right) \mathrm{e}^{\mathrm{j} k_{x} x} \mathrm{e}^{\mathrm{j} k_{y}} \mathrm{~d} k_{x} \mathrm{~d} k_{y} .
$$


Next, let us define the filter function $G^{(s p)}\left(k_{x}, k_{y}\right) \equiv \operatorname{III}\left(R k_{y}\right) H\left(k_{x}, k_{y}\right)$. Its superscript $(s p)$ indicates that the filter has two purposes: limiting the integration to the radiation disc and forcing the periodicity of the velocity field in the circumferential direction. If we denote the inverse Fourier transform of $G^{(s p)}\left(k_{x}, k_{y}\right)$ by $g^{(s p)}(x, y)$, the convolution theorem allows one to express the supersonic velocity as

$$
\bar{v}_{w}^{(s)}(x, y)=v_{w}(x, y) * g^{(s p)}(x, y),
$$

and the same holds true for the acoustic pressure,

$$
\bar{p}^{(s)}(x, y)=p(x, y) * g^{(s p)}(x, y) .
$$

The velocity field $v_{w}(x, y)$ can be directly obtained from the GEM described in Section 2.2, while the pressure field $p(x, y)$ is acquired from Section 2.3.1. All that is lacking to compute the supersonic velocity and pressure, and therefore the supersonic intensity, is an expression for $g^{(s p)}(x, y)$. This can be computed from the inverse Fourier transform,

$$
\begin{aligned}
\bar{g}^{(s p)}(x, y) & =\frac{1}{(2 \pi)^{2}} \int_{-\infty}^{+\infty} \int_{-\infty}^{+\infty} \operatorname{III}\left(R k_{y}\right) H\left(k_{x}, k_{y}\right) \mathrm{e}^{\mathrm{j} k_{x} x} \mathrm{e}^{\mathrm{j} k_{y}} \mathrm{~d} k_{x} \mathrm{~d} k_{y} \\
& =\frac{1}{(2 \pi)^{2} R} \sum_{n=-\infty}^{+\infty} \mathrm{e}^{\mathrm{j}(n / R) y} \int_{-\infty}^{+\infty} H\left(k_{x}, \frac{n}{R}\right) \mathrm{e}^{\mathrm{j} k_{x} x} \mathrm{~d} k_{x} \\
& =\frac{1}{(2 \pi)^{2} R} \sum_{n=\left\lceil-k_{0} R\right\rceil}^{\left\lfloor k_{0} R\right\rfloor} 2 \kappa_{x} \mathrm{e}^{\mathrm{j}(n / R) y} \operatorname{sinc}\left(\kappa_{x} x\right),
\end{aligned}
$$

where in the second line use has been made of the definition of Dirac's comb distribution in Eq. (28). In the third line, the integral is carried out and $\kappa_{x}=+\left[k_{0}^{2}-(n / R)^{2}\right]^{1 / 2}$. Given that $\kappa_{x}^{2}>0$, this implies $|n|<\left|k_{0} R\right|(\lfloor x\rfloor$ and $\lceil x\rceil$ respectively stand for the floor and ceil functions of argument $x)$. Note that $(n / R) y=n \theta$. The sinc function in Eq. (36) has an infinite amount of side lobes that in practice can pollute the solution. As quoted in [45], one can get rid of most unwanted ringing oscillations associated to the Gibbs phenomenon by resorting to a Lanczos filter. The function sinc $\left(\kappa_{x} x\right)$ is replaced with the product $\operatorname{sinc}\left(\kappa_{x} x\right) \operatorname{sinc}\left(\kappa_{x} x / \alpha\right)$ for $\kappa_{x} x \in(-\alpha, \alpha)$ and is set to zero otherwise. Typical values for the $\alpha$ parameter are $\alpha=2,3$, because integers make the sinc functions vanish at the edge of the filter, providing a smooth cut-off. Note that one could have arrived at Eqs. (34)-(36) by different paths, such as initially considering a Fourier series development for the radial velocity and acoustic pressure in the circumferential direction instead of using the Fourier transform and the convolution with the Dirac comb distribution. However, the procedure followed above has the advantage of allowing one to directly exploit the field values $v_{w}(x, y)$ and $p(x, y)$ from previous sections and it is also directly comparable to the spatial approach to supersonic intensity for flat plates in [45].

Once we have computed the supersonic velocity $\bar{v}_{w}^{(s)}(x, y)$ in Eq. (34) and the supersonic pressure $\bar{p}^{(s)}(x, y)$ in Eq. (35), the supersonic intensity can be obtained as

$$
I^{(s)}(x, y)=\frac{1}{2} \operatorname{Re}\left[\bar{p}^{(s)}(x, y) \bar{v}_{w}^{(\mathrm{s})}(x, y)^{*}\right],
$$

where the asterisk superscript denotes complex conjugate.

To compute the supersonic intensity for the ABH cylinder, we follow the strategy in the previous section where the cylinder was divided into $N$ radiators of area $\Delta S$. The convolution operation in Eqs. (34) and in (35) can be discretized for each radiator as,

$$
\begin{gathered}
v_{w}^{(s)}\left(x_{S}, y_{S}\right)=\Delta S \sum_{n=1}^{N} v_{w}\left(x_{n}^{\prime}, y_{n}^{\prime}\right) g^{(s p)}\left(x_{S}-x_{n}^{\prime}, y_{S}-y_{n}^{\prime}\right) \\
p^{(s)}\left(x_{S}, y_{S}\right)=\Delta S \sum_{n=1}^{N} p\left(x_{n}^{\prime}, y_{n}^{\prime}\right) g^{(s p)}\left(x_{S}-x_{n}^{\prime}, y_{S}-y_{n}^{\prime}\right) .
\end{gathered}
$$




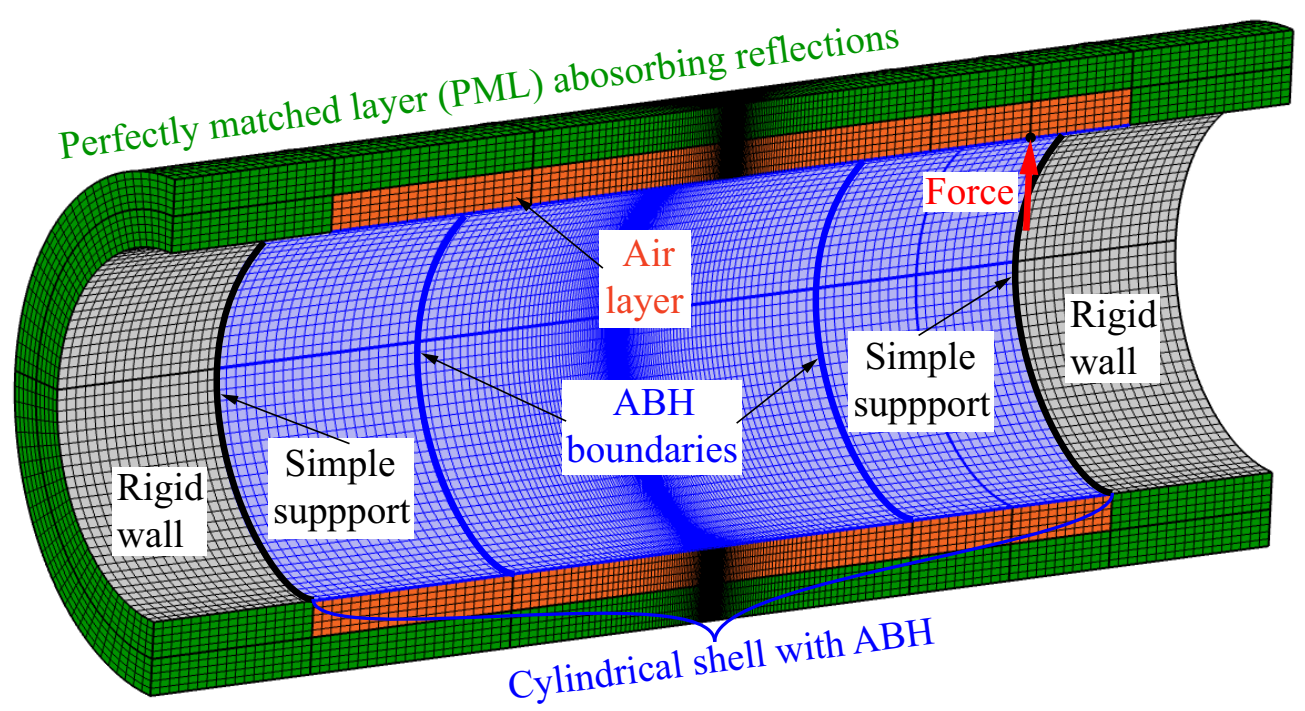

Figure 2: Sketch and mesh of the shell-acoustic coupled FEM model (3D sectional view) to validate the proposed approach for computing sound radiation from an annular ABH cylindrical shell. The blue mesh corresponds to the cylinder which is simply supported to the rigid baffles. The mesh is strongly refined at the central ABH region. An air layer (orange colour) is coupled to the cylinder and surrounded with a PML (green colour) to avoid reflection of outwards propagating acoustic waves.

where $\left(x_{S}, y_{S}\right)$ stands for the centre of the considered radiator. The supersonic intensity is finally recovered from,

$$
\mathbf{I}^{(s)}=\frac{1}{2} \operatorname{Re}\left[\overline{\mathbf{p}}^{(s)} \odot \overline{\mathbf{v}}_{w}^{(s)}\right],
$$

where $\odot$ indicates the Hadamard product (entrywise) between the supersonic pressure and complex conjugate supersonic velocity vectors.

\section{Model validation}

In this section, we validate the computations of the cylinder radial velocity, $v_{w}(x, y)$, performed with the GEM approach of Section 2.2 and the calculation of the acoustic wall pressure field, $p(x, y)$, obtained from the developments in Section 2.3.1. The validation is carried out by comparison with FEM simulations using

\begin{tabular}{cc}
\hline Geometry parameters & Material parameters \\
\hline$m=3$ & $\rho=7800 \mathrm{~kg} / \mathrm{m}^{3}$ \\
$a=2.5 \mathrm{~m}$ & $E=210 \mathrm{GPa}$ \\
$R=1 \mathrm{~m}$ & $\eta=0.005$ \\
$h_{\mathrm{uni}}=0.02 \mathrm{~m}$ & $\nu=0.3$ \\
$r_{\mathrm{abh}}=1.25 \mathrm{~m}$ & \\
$\varepsilon=0.0096 \mathrm{~m}^{-2}$ & $\rho_{v}=950 \mathrm{~kg} / \mathrm{m}^{3}$ \\
$h_{c}=0.0013 \mathrm{~m}$ & $E_{v}=5 \mathrm{GPa}$ \\
$r_{v}=1.25 \mathrm{~m}$ & $\eta_{v}=0.5$ \\
$h_{v}=0.008 \mathrm{~m}$ & $\nu_{v}=0.3$ \\
\hline
\end{tabular}

Table 1: Geometry and material parameters of the ABH cylindrical shell. $\rho$ : shell density, $\rho_{v}$ : damping layer density, $E$ : shell Young modulus, $E_{v}$ : damping layer Young modulus, $\eta$ : shell loss factor, $\eta_{v}$ : damping layer loss factor, $\nu$ : shell Poisson ratio, $\nu_{v}$ : damping layer Poisson ratio. 


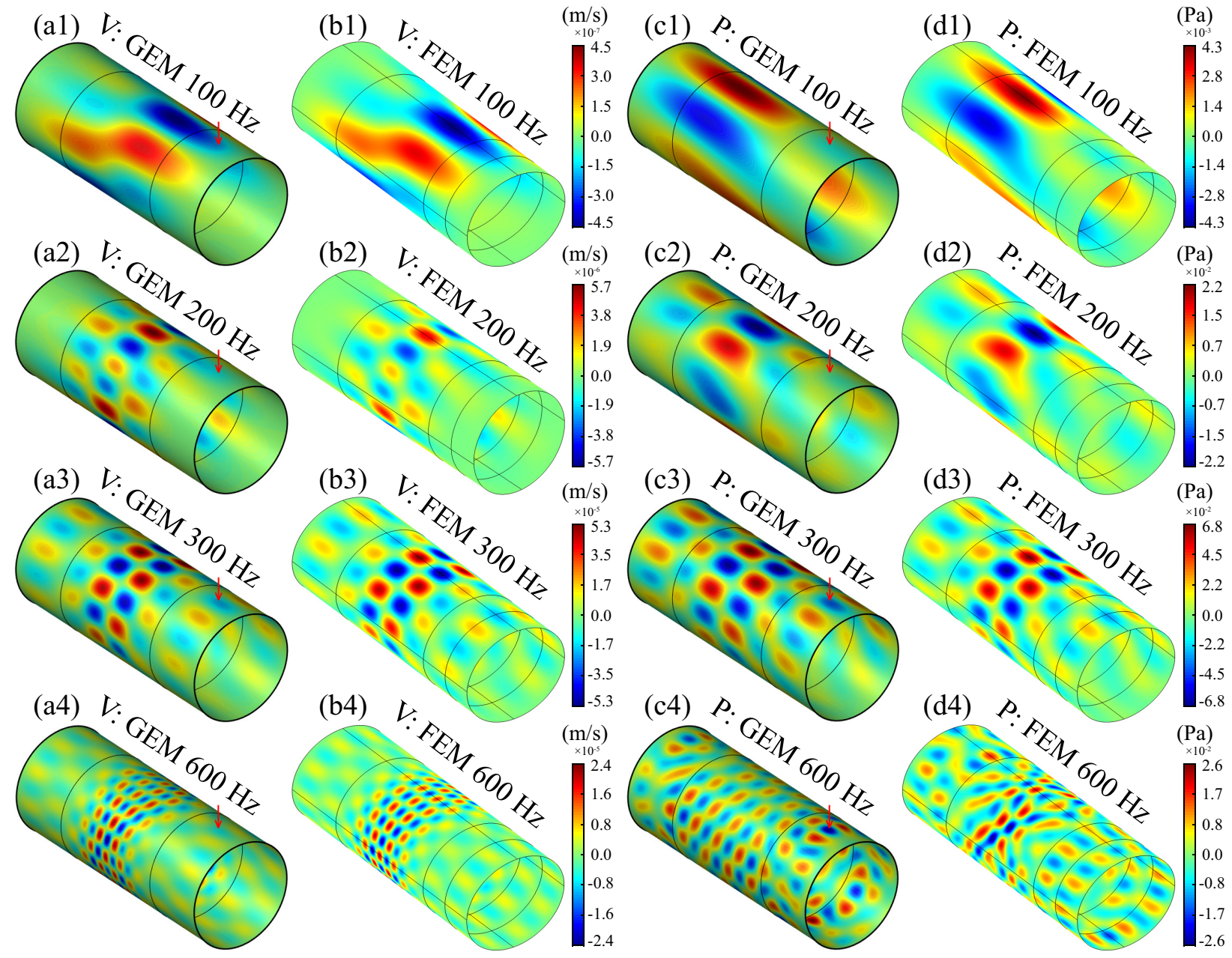

Figure 3: Validation of the proposed GEM plus sound radiation approach against FEM simulations. Left columns: surface radial velocity, $v_{r}(x, y)$, indicated as $\mathrm{V}$ in the figure. Right columns: acoustic wall pressure, $\mathrm{p}(\mathrm{x}, \mathrm{y})$, indicated as $\mathrm{P}$ in the figure. Results corresponding to the annular ABH cylindrical shell under a unit point excitation (red arrow in the figures) at frequencies $100,200,300$ and $600 \mathrm{~Hz}$.

the commercial package COMSOL Multi-physics. As regards the GEM model, we have taken $s_{x}=4$ and $s_{y}=3$ which results in 89 Gaussian functions in the $x$ direction and 55 in the $y$ direction. Consequently, the mass and stiffness matrices in Eq. (19) have dimensions 14,685 × 14,685. For the computation of the surface acoustic pressure of the shell through Eq. (22), we have considered a discretization of $100 \times 100$ patches.

In what concerns the FEM model, the 3D shell-acoustic coupling module of COMSOL has been selected. The annular ABH is simulated in FEM by prescribing a local thickness of the shell according to the powerlaw introduced in Section 2.1 (see the blue mesh in Fig. 2). Given the symmetry of the model, we have just considered half of the cylinder for the simulations and applied symmetric boundary conditions to reduce the computational cost. The two ends of the cylindrical shell are connected to rigid baffles by means of simple supports. The rigid baffles of length $L_{\mathrm{baf}}=1.0 \mathrm{~m}$ at both sides of the cylinder are intended to approximate the ideal infinite ones in Fig. 1a. Outside the shell, an air layer (orange color in Fig. 2) of thickness $h_{a}=0.2 \mathrm{~m}$ is coupled to the ABH shell surface. To avoid reflected sound waves, a perfectly matched layer (PML) of thickness $h_{\mathrm{pml}}=0.2 \mathrm{~m}$ (green color in Fig. 2) surrounds the air layer. The whole FEM model has been meshed using hexahedral (for air) and quadrilateral (for shell) elements. In the rigid walls and the uniform portions of the shell, the mesh size is $h \sim 0.06 \mathrm{~m}$. However, a very fine mesh is needed 
to capture the rapid wavelength changes at the central region of the ABH portion. Therefore, the mesh has been gradually decreased towards the $\mathrm{ABH}$ centre to $1 / 15$ of its size in the uniform part (see Fig. 2). The whole FEM model consists of 106,380 elements (including 8,802 shell elements for the ABH cylinder) and 119,790 nodes, with a total of 1,118,013 degrees of freedom. On the other hand, all material and geometrical parameters corresponding to the annular ABH cylindrical shell sketched in Fig. 1 are reported in Table 1. The harmonic unit point force is radially exerted at $x_{f}=1.875 \mathrm{~m}$.

In Fig. 3 we present a comparison between the shell surface radial velocity and the acoustic wall pressure computed with both the GEM plus radiation model, and the FEM. The results are presented for the frequency values 100, 200, 300 and $600 \mathrm{~Hz}$. The left two columns (Figs. 3a1-3a4 and Figs. 3b1-3b4) show the surface velocity distribution, $v_{w}(x, y)$, whereas the two columns in the right (Figs. 3c1-3c4 and Figs. 3d1-3d4) exhibit the acoustic wall pressure distribution, $p(x, y)$. As observed from the left column, a close matching exists between the radial surface velocity from the proposed method and that of FEM (see also the deeper validation of the GEM for annular ABH cylinder vibrations in [30]). As for the sound radiation in the right columns, the results are very similar to those of FEM for the first three frequencies, namely 100,200 and $300 \mathrm{~Hz}$ (compare Figs. 3c1-3c3 with Figs. 3d1-3d3). However, strong differences manifest at $600 \mathrm{~Hz}$ (see Figs. 3c4 and3d4). These are mostly attributed to two facts. First, the difference in the acoustic boundary conditions used for the FEM and GEM plus integral radiation approach. While semi-infinite baffles are adopted for the formulation in Eq. (20) (see the black shell in Fig. 1a), only baffles of a finite extent can be simulated with FEM (see the rigid walls in Fig. 2). Second, the patch discretization may introduce some errors at high frequencies. Remember that we have taken 100 patches in the axial direction and if one assumes the six patch per wavelength criteria, the model is valid up to $f=1,143 \mathrm{~Hz}$, but a slight refinement of 10 patches per wavelength would diminish the threshold frequency to $f=686 \mathrm{~Hz}$. A finer mesh is beyond the capabilities of the computer used for the calculations in this work so it is logical that some discrepancies manifest close to and beyond $600 \mathrm{~Hz}$. Despite such differences in acoustic wall pressure found at higher frequencies, which are credited to patch number limitation and distinct boundary conditions between models, rather than to theoretical limitations, it is apparent that the proposed approach to compute the sound radiated from annular ABH cylindrical shells is reliable for the considered frequency range, and for the purposes in this paper.

\section{Simulation results}

\subsection{Near-field acoustic characteristics}

\subsubsection{Sound power level reduction and radiation efficiency}

In this section, we explore how embedding an annular ABH on the cylindrical shell affects quantities such as its sound power level and radiation efficiency and therefore helps reducing sound emission. Before that, however, let us recall the expressions for the critical frequency, $f_{c}$, and the ring frequency, $f_{R}$, of cylindrical shells [32],

$$
\begin{gathered}
f_{c}=\frac{c_{0}^{2}}{2 \pi h(x)} \sqrt{\frac{12 \rho\left(1-\nu^{2}\right)}{E}}, \\
f_{R}=\frac{1}{2 \pi R} \sqrt{\frac{E}{\rho\left(1-\nu^{2}\right)}} .
\end{gathered}
$$

In the case of flat plates, flexural wave propagation speed equals the speed of sound in air at the critical frequency and the radiation efficiency, $\sigma$ in Eq.(25), strongly peaks at this value. Below $f_{c}$, flexural waves are subsonic and barely radiate outwards because of acoustic recirculation on the radiating surface. Therefore, radiation basically takes place at plate boundaries. In contrast, once passed the critical frequency, $f>f_{c}$, the radiation efficiency tends to unity and acoustic waves are effectively radiated outwards. $f_{c}$ also plays a marked role for cylindrical shell radiation in combination with $f_{R}$. As explained in [32] and commented 


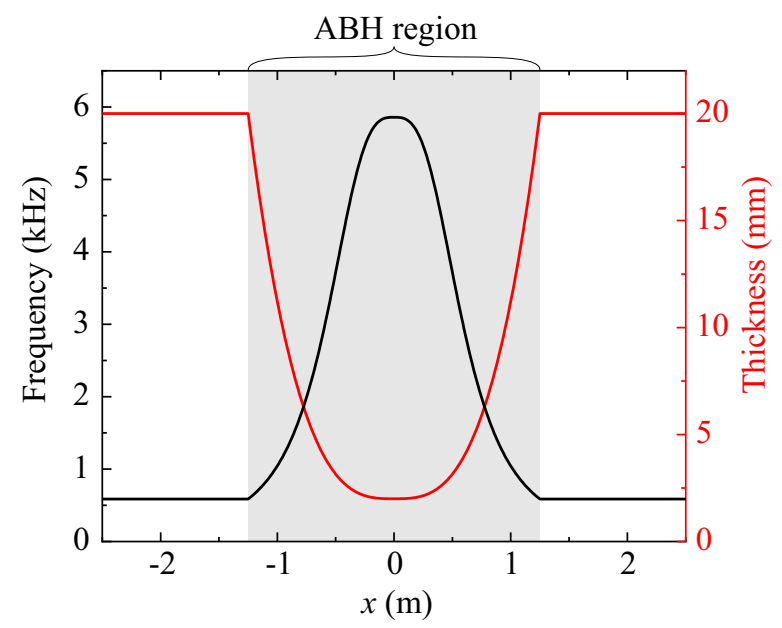

Figure 4: Dependence of critical frequency $f_{c}$ on location (black curve) together with thickness variation profile (red curve). Transonic boundaries $x_{t}$ are obtained by intersecting a constant frequency line with the $f_{c}$ curve.

in the Introduction, for thin acoustic shells, i.e. $f_{R} / f_{c}<1$, the radiation efficiency follows a general trend. It increases with frequency up to $f_{R}$; then, once surpassed $f_{R}$, curvature effects loose importance and $\sigma$ resembles that of a flat plate. Between $f_{R}<f<f_{c}$, the radiation efficiency first diminishes and then starts increasing again until $f_{c}$ is reached. After the critical frequency, i.e., for $f>f_{c}, \sigma$ tends to unity. However, for thick acoustic cylindrical shells in which $f_{R} / f_{c}>1$ the situation is far more complex and $\sigma$ depends on the type of excitation and on the geometrical and physical parameters of the cylindrical shell as well [32]. In the present example, the uniform cylinder without ABH (see Table 1), corresponds to a thick acoustic shell with $f_{R}=865 \mathrm{~Hz}$ and $f_{c}=585 \mathrm{~Hz}$ (as said in the Introduction that has nothing to do with the thin shell theory assumptions valid whenever $h_{\text {uni }} \ll R$; a shell can be thin and acoustically thick as in the current example). Yet, it follows from Eqs. (41) and (42) that while $f_{R}$ is constant, $f_{c}$ depends on the shell thickness. As a consequence, when embedding the ABH on the cylinder one can no longer assign a single value to $f_{c}$ since that would increase inside the $\mathrm{ABH}$ until a maximum value was attained for the central $\mathrm{ABH}$ truncation thickness. In the present case, the critical frequency for a uniform shell having the truncation thickness $h_{c}$ in Table 1 would be $f_{c}=6 \mathrm{kHz}$ and therefore correspond to a thin acoustic shell instead of a thick one. So what does occur inside the ABH? It is expected that structural supersonic waves propagating in the axial direction will progressively slow down when approaching the ABH centre and eventually become subsonic once passed a transonic boundary. In Fig. 4, we have plotted the dependence of $f_{c}$ with position (the grey shaded area stands for the $\mathrm{ABH}$ region) together with the variation profile. For a fixed frequency value, its intersection with the critical frequency curve determines the location of the transonic boundaries. This phenomenon will be explained in more detail in the forthcoming section.

Apart from the critical and ring frequencies, two other important frequencies for $\mathrm{ABHs}$ are the radius cut-on frequency $f_{r_{\mathrm{abh}}}=38 \mathrm{~Hz}$ and the smoothness cut-on frequency $f_{\varepsilon}=5 \mathrm{~Hz}$. These two frequencies govern the functioning of ABHs on flat plates (see e.g., $[2,38]$ ). Actually the ABH effect is expected to be fully operative beyond $3 f_{\varepsilon}=15 \mathrm{~Hz}$ and usually it turns out $f_{\mathrm{r}}<f_{\varepsilon}$. Nonetheless and as quoted in [30], for $\mathrm{ABH}$ cylindrical shells $f_{c}$ and $f_{R}$ play a much more important role than the $\mathrm{ABH}$ cut-on frequencies because the annular $\mathrm{ABH}$ is designed to influence flexural motion, while the circumferential one often dominates the lower part of the spectrum.

Let us start examining the surface mean square velocity (MSV $=10 \log _{10}\left\langle v_{w}^{2}\right\rangle_{S}$ ) of the cylindrical shell with and without $\mathrm{ABH}$ and parameters in Table 1, as well as its corresponding sound power levels (SWL $=10 \log _{10}\left(W_{\text {rad }} / W_{0}\right)$, with $W_{\text {rad }}$ obtained from Eq. (24) and $W_{0}=10^{-12}$ Watts). As seen in Fig. 5 , the MSV and SWL of the ABH shell are considerably lower than those of the uniform shell. As regards the reduction in MSV of Fig. 5a, the ABH functions very well beyond the critical frequency $f_{c}=585 \mathrm{~Hz}$, because supersonic 

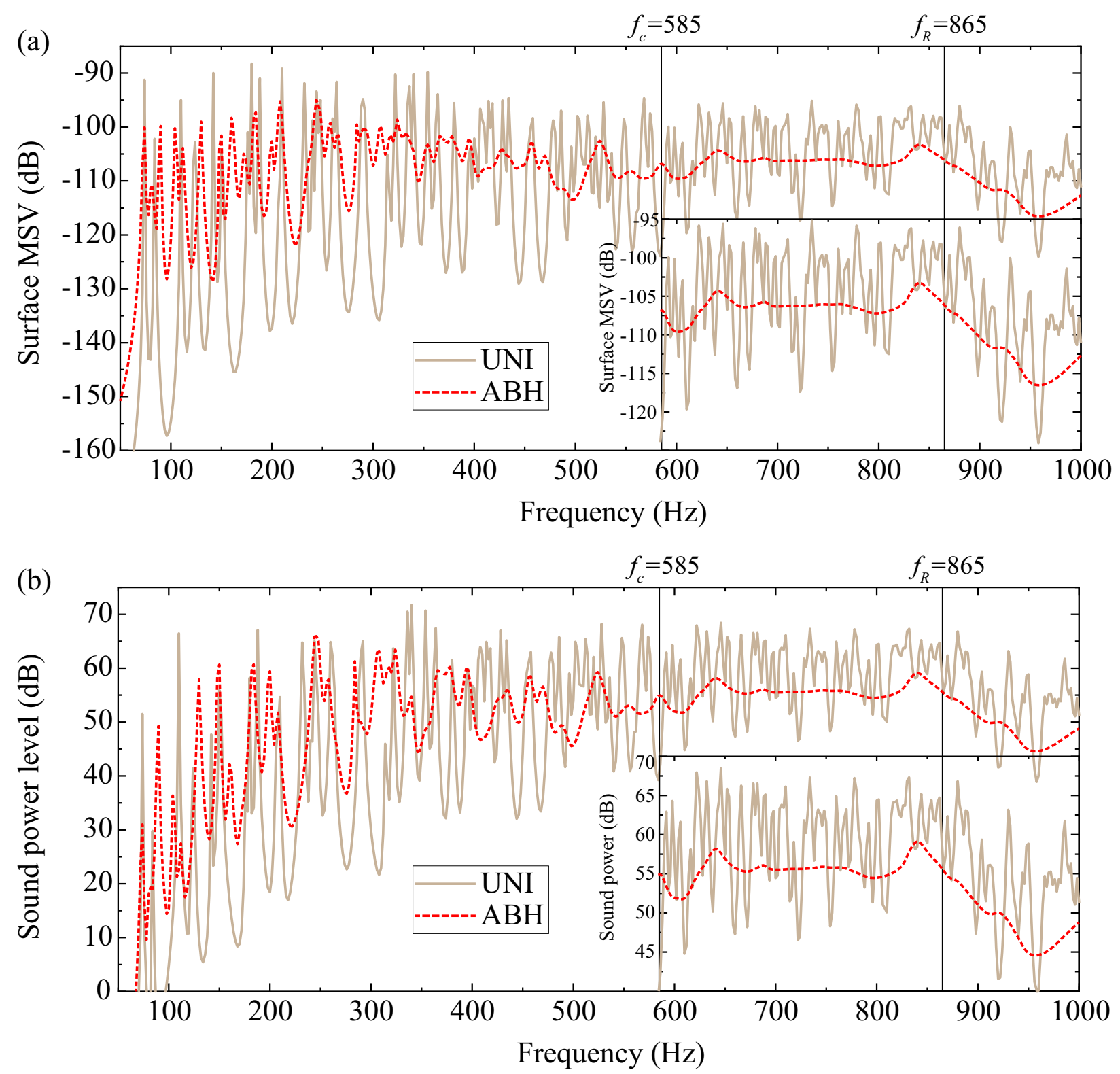

Figure 5: Comparison between the performance of a uniform cylindrical shell and a shell with an embedded annular ABH (a) mean squared velocity (MSV) and (b) sound power level (SWL).

flexural motion becomes subsonic and dissipated by the damping layer inside the ABH. A zoom-in window to better appreciate the performance of the $\mathrm{ABH}$ in that frequency range has been included in the figure showing that, in average, the MSV diminishes about $15 \mathrm{~dB}$. It is also observed in the figure that the MSV for both the uniform and ABH shells clearly decreases above the ring frequency $f_{R}=865 \mathrm{~Hz}$. Below that frequency the curvature of the cylinder plays a central role and the motions in the axial, circumferential and radial directions are strongly coupled. As said before, when $f>f_{R}$, the shell behaviour starts to resemble that of a flat plate and the coupling between different types of waves gets much weaker. Some more insight on this point will be provided in next subsection. On the other hand, at lower frequencies, i.e., for $f \ll f_{c}$ the ABH shell MSV is also considerably lower than that of the uniform shell (about $10 \mathrm{~dB}$ in average). That reduction is attributed to the smaller rigidity of the $\mathrm{ABH}$ shell due to the $\mathrm{ABH}$ indentation rather 


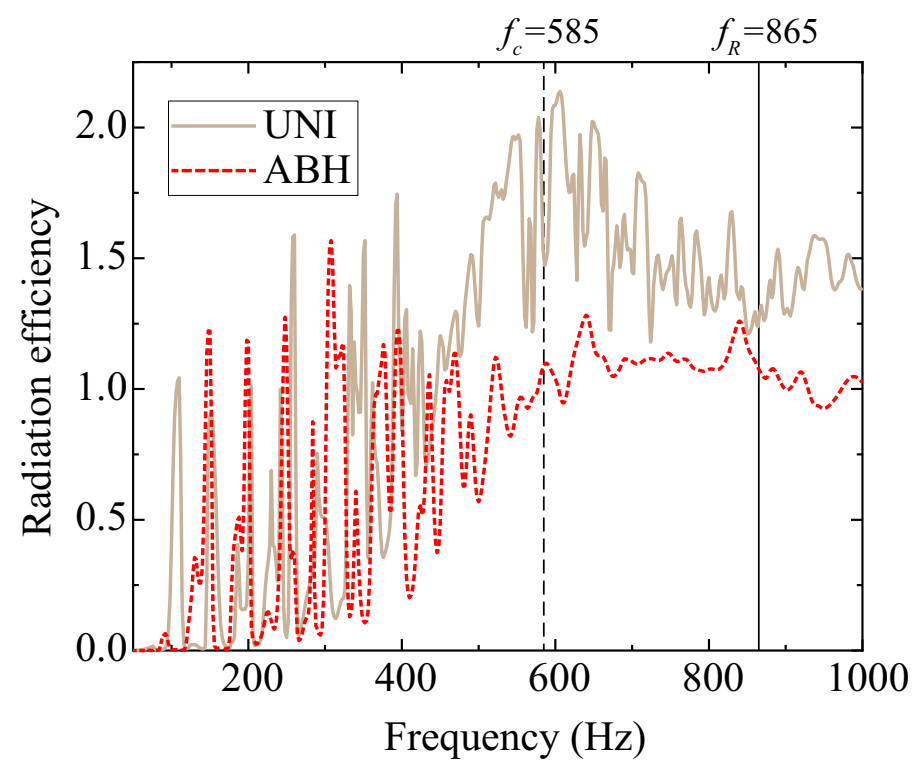

Figure 6: Radiation efficiency, $\sigma$, for a uniform cylindrical shell and for a shell with an embedded annular ABH.

than to the ABH effect. Very similar trends to those of the MSV are recovered for the SWL in Fig. 5b. For frequencies greater than $f=320 \mathrm{~Hz}$, reductions up to $10 \mathrm{~dB}$ are achieved throughout the spectrum. The explanations for the SWL performance are essentially those reported for the MSV, and a zoom is also included in the figure to better observe the benefits attained for $f>f_{c}$. This strong reduction in SWL values is a first indication that one may achieve a significant decrease in the radiated sound by embedding an annular $\mathrm{ABH}$ on a cylindrical shell. That is reinforced having a look at the radiation efficiency in Fig. 6. For the uniform shell in this example, it is seen that $\sigma$ increases from negligible values at low frequencies to a peak value of $\sim 2$ close to $f_{c}$. Then it diminishes again until $f_{R}$ is reached and $\sigma$ becomes more or less stable for $f>f_{R}$, in the analysed frequency range. In the figure, one can clearly appreciate how the annular $\mathrm{ABH}$ indentation is capable of totally suppressing the peak value at the critical frequency, keeping it close to 1 instead of 2. $\sigma$ becomes almost constant and equal to 1 for most frequencies between $f_{c}<f<f_{R}$ and then slightly decreases once surpassed $f_{R}$.

\subsubsection{Source location through supersonic intensity}

To determine which regions on the surface of the uniform and $\mathrm{ABH}$ cylindrical shells most contribute to the radiation of acoustic pressure to the far field, we resort to the supersonic intensity (SSI) introduced in Section 2.3.2. For comparison, results for standard surface acoustic intensity (hereafter referred to as sonic intensity: SI) are also presented. In Fig. 7, the values of SI and SSI for ABH and uniform shells have been plotted at the four different frequencies $f_{1}=110 \mathrm{~Hz}, f_{2}=340 \mathrm{~Hz}, f_{3}=778 \mathrm{~Hz}$ and $f_{4}=930 \mathrm{~Hz}$. The left column in the figure, Figs. 7a1-a4 shows the SI values for the ABH shell while those of SSI are plotted in Figs. 7b1-7b4. Likewise, Figs. 7c1-7c4 present the SI for the uniform shell, while the SSI is provided in the right column, Figs. 7d1-7d4. In each figure, the cylinder has been mapped into a flat surface for easier inspection. The horizontal axis corresponds to the axial direction and the vertical one to the circumferential direction. The colour scales change from figure to figure to better perceive their contents. The cross in each plot indicates the point where the external force is applied and the two vertical lines denote the ABH limits.

Let us first focus on the outcomes from the lowest frequency in the figure, $f_{1}=110 \mathrm{~Hz}$ (first row in Fig. 7). As observed for the ABH shell in Fig. 7a1, SI mostly concentrates within the ABH exhibiting positive and negative values which result in very poor radiation efficiency. Its corresponding SSI in Fig. 7b1 is basically positive but its maximum value reaches $5.8 \times 10^{-11} \mathrm{~W} / \mathrm{m}^{2}$, which is much lower than the maximum SI $\left(1.6 \times 10^{-8} \mathrm{~W} / \mathrm{m}^{2}\right)$. Moreover, the SSI pattern is completely different indicating that most radiated sound 

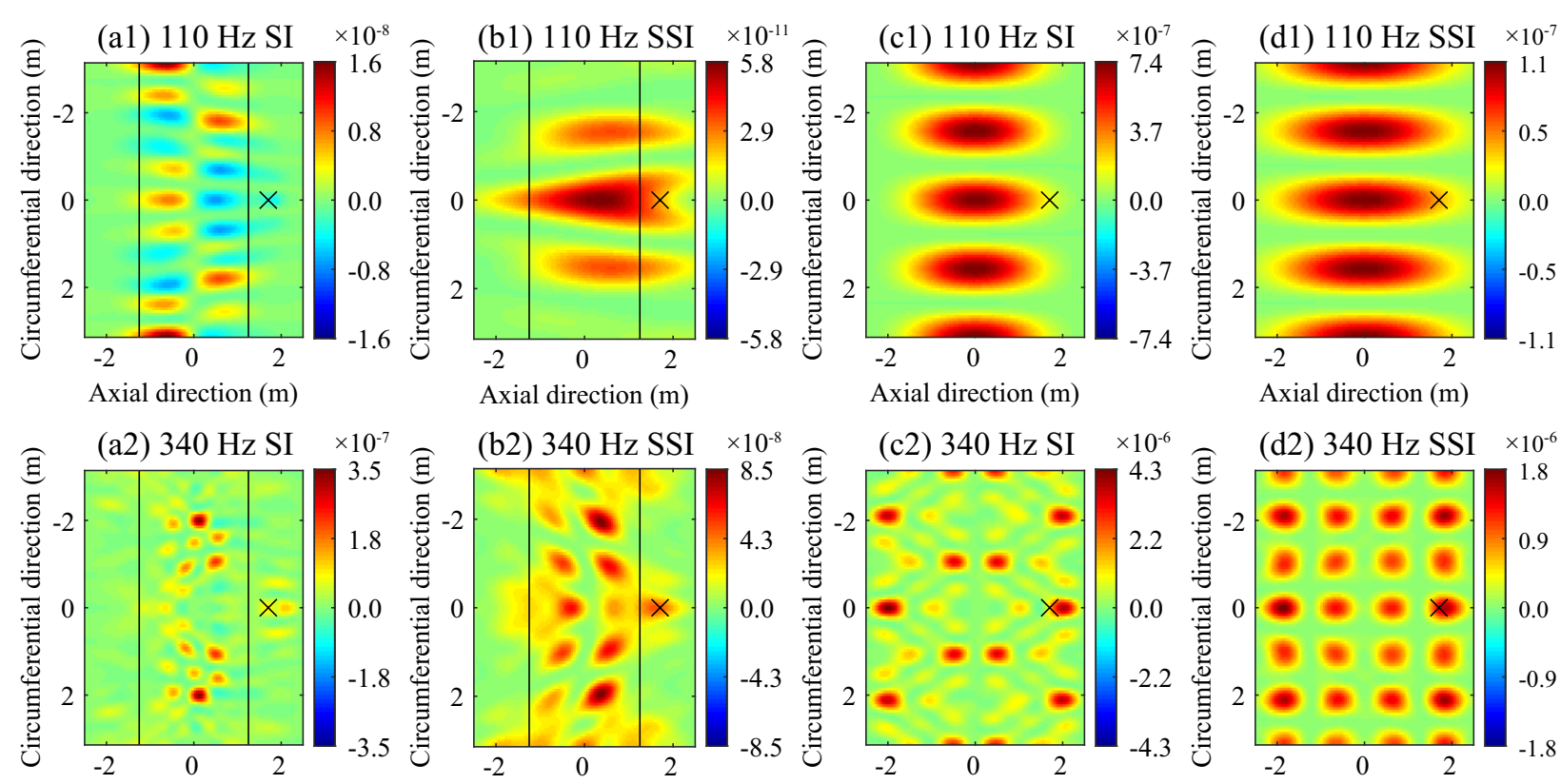

(b2) $340 \mathrm{~Hz}$ SSI $\times 10^{-8}$
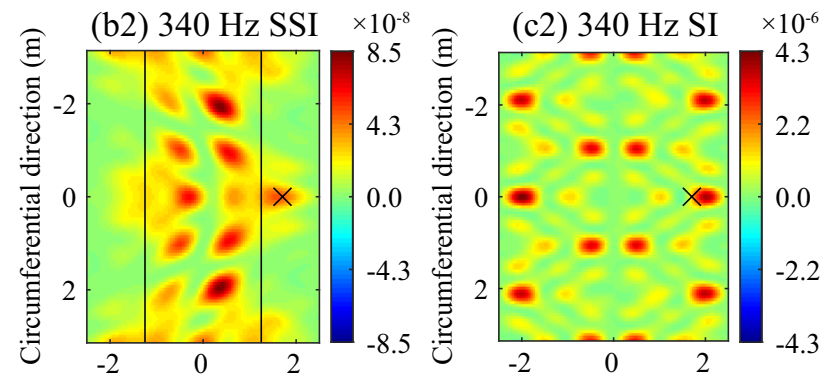

(d2) $340 \mathrm{~Hz} \mathrm{SSI} \times 10^{-6}$

Axial direction $(\mathrm{m})$

Axial direction (m)

Axial direction (m)

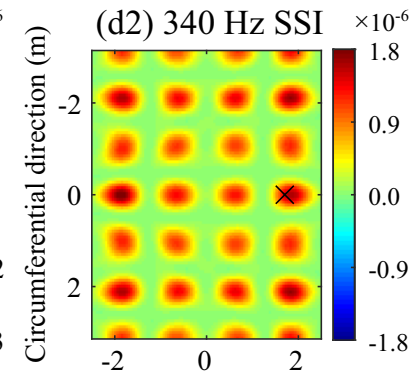

(b3) $778 \mathrm{~Hz} \mathrm{SSI} \times 10^{-7}$
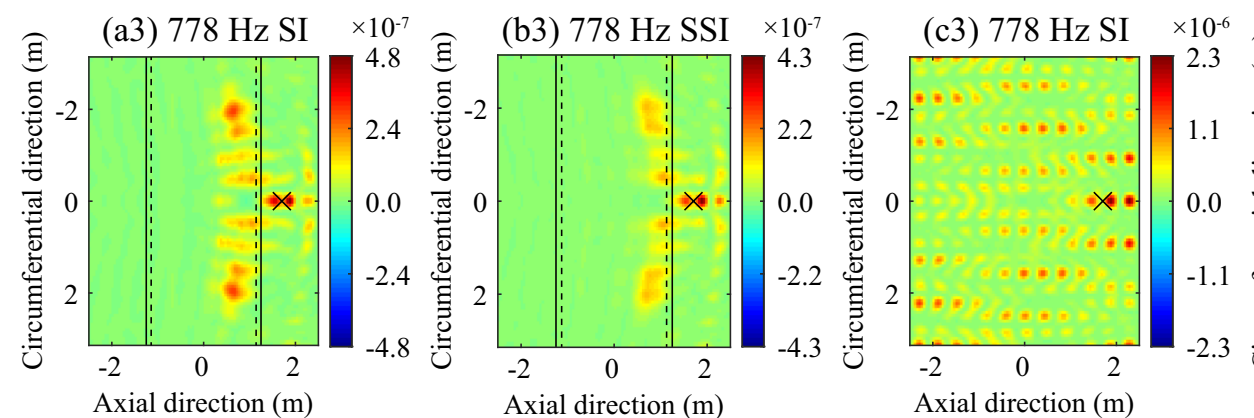

Axial direction (m)

Axial direction (m)
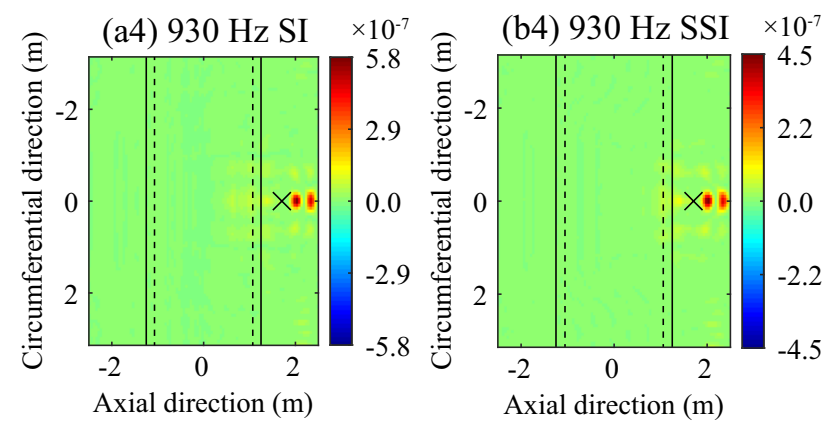

Axial direction (m)
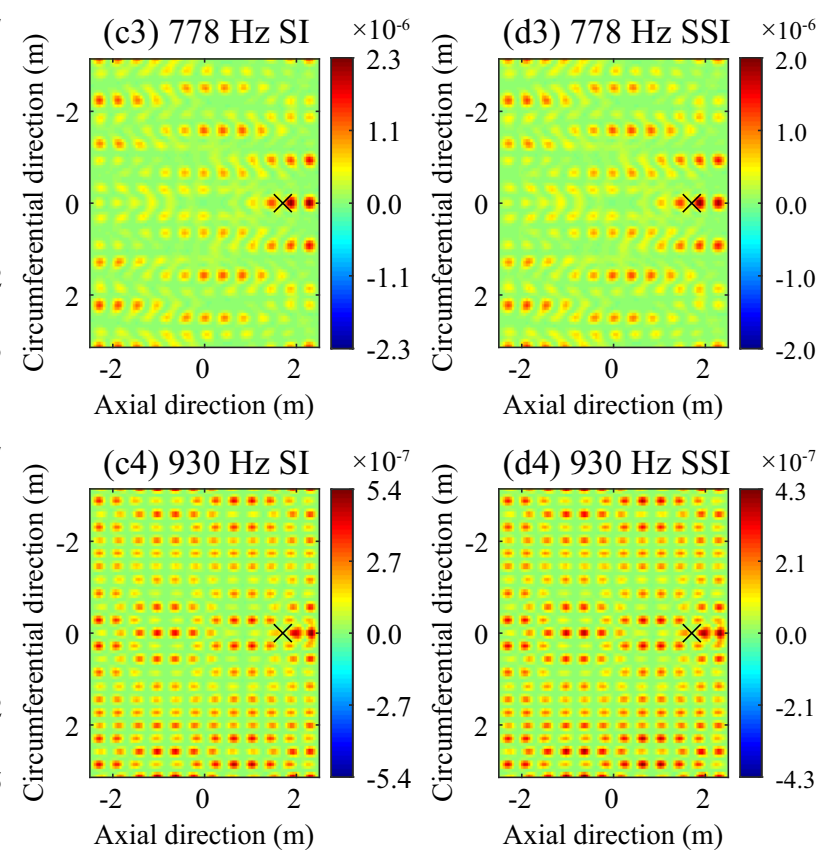

Figure 7: Sonic (SI) and supersonic (SSI) intensity $\left(\mathrm{W} / \mathrm{m}^{2}\right)$ for frequencies $f_{1}=110 \mathrm{~Hz}, f_{2}=340 \mathrm{~Hz}, f_{3}=778 \mathrm{~Hz}$ and $f_{4}=930 \mathrm{~Hz}$. Column (a): SI for the ABH shell, Column (b): SSI for the ABH shell, Column (c): SI for the uniform shell, Column (d): SSI for the uniform shell

emanates along the top of the cylinder, where excitation takes place, and from its sides, but not from the bottom area. One may wonder why is it that so much SSI is generated within the ABH. The reason is that for such low frequency, $f_{1} \ll f_{R}$, curvature effects are very important and the shell is partially stiffened because of it, compensating the weakening caused by the ABH indentation. Circumferential supersonic modes dominate the shell behaviour $[5,6,30]$ and the $\mathrm{ABH}$ has very little effect on them because it is 

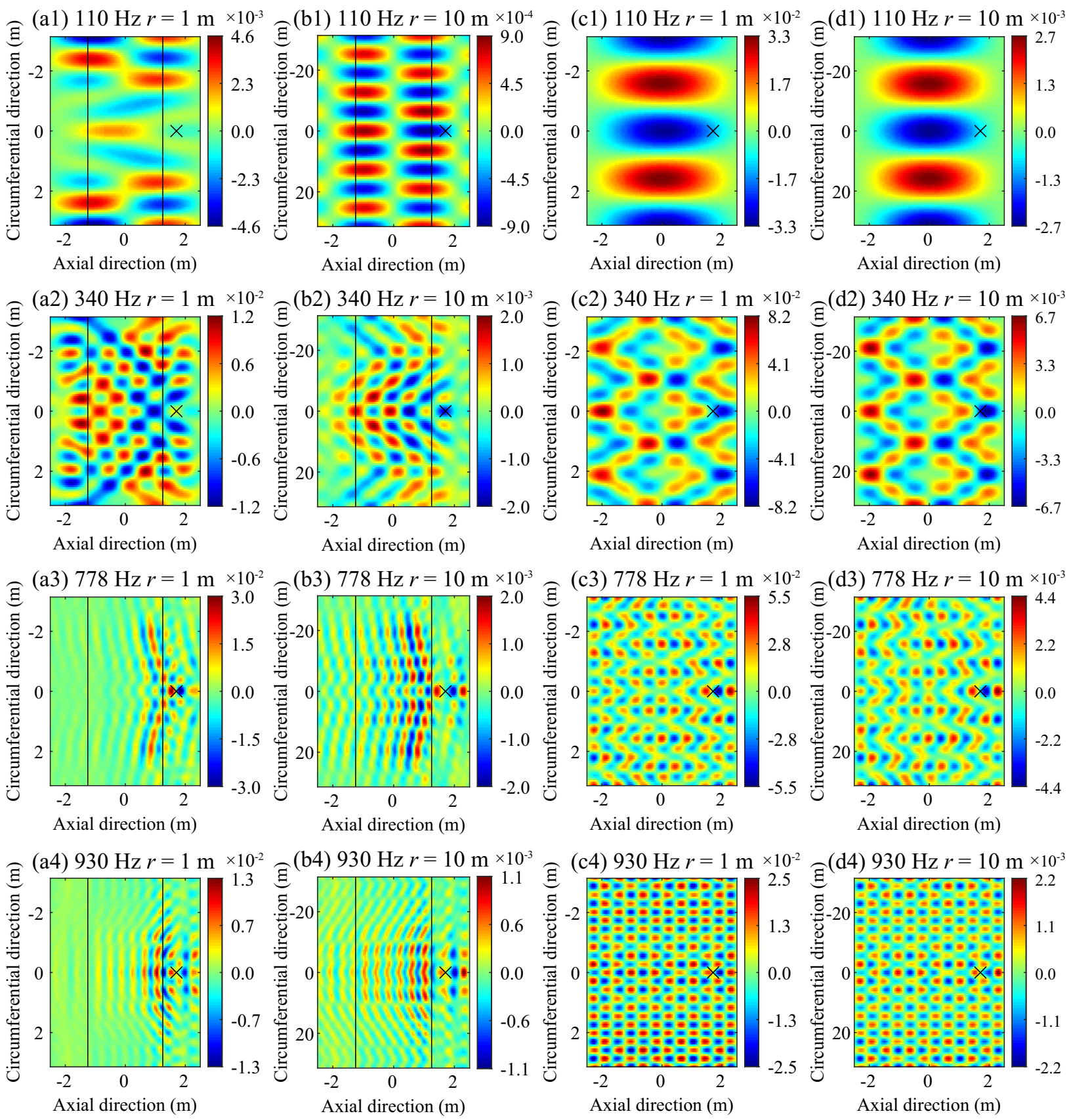

Figure 8: Surface and far-field acoustic pressure $(\mathrm{Pa})$ for frequencies $f_{1}=110 \mathrm{~Hz}, f_{2}=340 \mathrm{~Hz}, f_{3}=778 \mathrm{~Hz}$ and $f_{4}=930 \mathrm{~Hz}$. Column (a): Surface pressure $(R=1)$ for the ABH shell, Column (b): Far-field pressure $(r=10)$ for the ABH shell, Column (c): Surface pressure $(R=1)$ for the uniform shell, Column (d): Far-field pressure $(r=10)$ for the uniform shell.

designed to act on waves propagating in the axial direction. This is confirmed having a look at the SI and SSI for the uniform shell in Fig. 7c1 and Fig. 7d1 in which circumferential motion predominates. Note that at this low frequency the SI and SSI of the uniform shell present very similar patterns.

If we move to the second analysed frequency, $f_{2}=340 \mathrm{~Hz}$, which is again considerably lower than the critical and ring frequencies $f_{c}$ and $f_{R}$, it is observed that negative SI values still appear in the annular ABH 
of Fig. 7a2, though these are smaller than those encountered for $f_{1}$. The SSI in Fig. $7 \mathrm{~b} 2$ still concentrates within the $\mathrm{ABH}$, with a pattern that now comprises the whole cylinder circumference. Its value is much higher than that found for $f_{1}$ indicating that more sound will be radiated outwards for $f_{2}$ than for $f_{1}$. In what concerns the uniform cylinder, it is seen in Fig. $7 \mathrm{c} 2$ that SI exhibits a regular combination of regions of maxima and minima which all become radiators in the figure for SSI (see Fig. 7d2).

The next frequency $f_{3}=778 \mathrm{~Hz}$ lies between $f_{c}$ and $f_{R}$ and reveals some additional interesting phenomena. In this case, transonic boundaries appear within the $\mathrm{ABH}$ in which supersonic flexural waves entering the $\mathrm{ABH}$ become subsonic as a result of the decrease in propagation speed. Transonic boundaries are indicated with dashed lines in Fig. 7 and consist of rings for the annular ABH, while become circles for circular ABHs in flat plates, see [4]. For a given incident wave with frequency $f>f_{c}$, the transonic boundary appears at the axial positions $\pm x_{t}$ where the thickness $h\left(x_{t}\right)$ is such that the critical frequency in Eq. (41) matches with the incident one $f$, i.e.,

$$
x_{t}=\left[\frac{c_{0}^{2}}{2 \pi \varepsilon f} \sqrt{\frac{12 \rho\left(1-\nu^{2}\right)}{E}}-\frac{h_{c}}{\varepsilon}\right]^{\frac{1}{m}} .
$$

This value is directly obtained combining Eq. (41) with the expression for the ABH thickness in section 2.1. As explained before, the location of the transonic boundaries $\pm x_{t}$ for a given frequency $f$ can be graphically obtained by intersecting the line of constant $f$ with the curve for the critical frequency in Fig. 4 . It is clearly seen in the figure that $f_{c}$ starts increasing as approaching the $\mathrm{ABH}$ centre, indicating the transonic boundaries gradually approach one to another at high frequencies.

Having a look at Fig. 7a3, it is observed that SI now mainly locates at the right part of the ABH shell, close to the excitation point. SI is also found at the interior of the $\mathrm{ABH}$ but one can see that the $\mathrm{ABH}$ effect is already significant and the SI values are small therein. The SSI pattern in Fig. 7b3 closely resembles that in Fig. 7a3 but the SSI gets smaller than SI once passed the transonic boundary, for the reasons explained before. Besides, note that the maximum SSI value outside the $\mathrm{ABH}$ is $4.3 \times 10^{-7} \mathrm{~W} / \mathrm{m}^{2}$ which is very similar to the SI one, $4.8 \times 10^{-7} \mathrm{~W} / \mathrm{m}^{2}$. This is logical since the radiation efficiency is high above the critical frequency and the main radiation spot is located in the neighbourhood of the excitation point. In what concerns the values and patterns for the SI and SSI in Figs. 7c3-7d3 they are very similar one to another and clearly higher than the values obtained for the $\mathrm{ABH}$ shell, indicating that the latter can be very effective in reducing sound radiation.

Finally, at the fourth frequency $f_{4}=930 \mathrm{~Hz}$, which is greater than the ring frequency $f_{R}=865 \mathrm{~Hz}$, the ABH becomes fully operative and almost no SSI is found inside the ABH once surpassed the transonic boundary (see Fig. 7b4), while some appears for the SI in Fig. 7a4. As regard the uniform shell in Figs. 7c4$7 \mathrm{~d} 4$, the values and patterns for the SI and SSI are again very similar because beyond the critical frequency all propagating flexural waves are supersonic.

\subsection{Far-field sound radiation}

Once seen how the annular ABH is capable of reducing the cylindrical shell sound power level and its radiation efficiency, and once determined the location of sources responsible for sound radiation by means of supersonic intensity, it is time to inspect how the acoustic far field becomes influenced by the ABH. To that purpose, we first consider the acoustic pressure distribution on a virtual cylindrical surface of radius $r=10 \mathrm{~m}$, at the same frequencies analysed in the previous subsection, namely $f_{1}=110 \mathrm{~Hz}, f_{2}=340 \mathrm{~Hz}$, $f_{3}=778 \mathrm{~Hz}$ and $f_{4}=930 \mathrm{~Hz}$. Results are presented in Fig. 8 together with the acoustic pressure at the cylinder surface, $R=1 \mathrm{~m}$, for comparison. Each row in the figure corresponds to one frequency. The two columns in the left contain the acoustic pressure distribution for the ABH shell at $R=1$ and $r=10$, respectively, while the columns in the right show the outcomes for the uniform shell at the same distances. If one compares the far field results $(r=10 \mathrm{~m})$ for the $\mathrm{ABH}$ and uniform shells, i.e., Figs. 8b1-8b4 with Figs. 8d1-8d4, one can clearly appreciate that embedding the annular ABH indentation on the cylinder reduces the emitted sound (note that the colour scales are different in the figures to better perceive their details). On the other hand, if one contrasts the surface acoustic pressure of the uniform shell (Figs. 8c1$8 \mathrm{c} 4$ ) with that at $r=10 \mathrm{~m}$ (Figs. 8d1-8d4) the same exact pattern is observed. That is, the figures only 

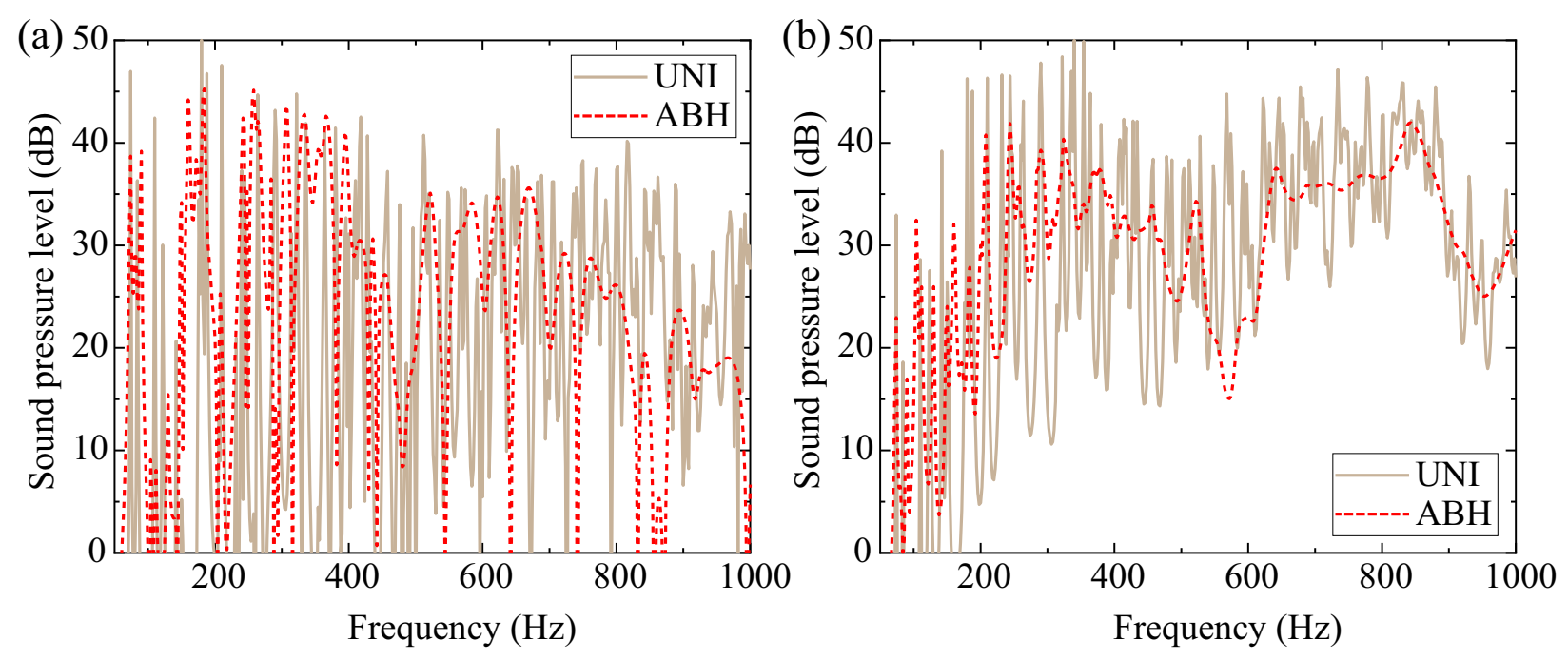

Figure 9: Far-field sound pressure level spectra at $r=10 \mathrm{~m}$ or points: $(\mathrm{a}) P 1=(0,0,10) ;(\mathrm{b}) P 2=(1.875,0,10)$. The coordinates $(x, y, r)$ refer to Fig. 8.

differ by a constant value due to geometrical divergence. As opposed, the surface patterns of the ABH shell (Figs. 8a1-8a4) are clearly distinct from those at the far field (Figs. 8b1-8b4).

To better recognize the role of the annular ABH, in Fig. 9 we have plotted the acoustic spectra at two far field points $P 1=(0,0,10)$ and $P 2=(1.875,0,10)$ according to the coordinates $(x, y, r)$ in Fig. 8 . Note that $P 2$ is localized in the vertical of the excitation point source. Fig. 9a shows the sound pressure level $\left(\mathrm{SPL}=20 \log _{10}\left(p / p_{0}\right), p_{0}=2 \times 10^{-5} \mathrm{~Pa}\right)$ at $P 1$ for the $\mathrm{ABH}$ and cylindrical shells. As observed, the SPL starts being attenuated for $f>400 \mathrm{~Hz}$ and becomes really effective for $f>700$, reaching reduction values of $20 \mathrm{~dB}$. At low frequencies, though, the benefits are not so clear because, as commented before, the $\mathrm{ABH}$ does not work properly below the ring frequency, where circumferential motion becomes important. In what concerns the second test point, $P_{2}$, the ABH improvement is very significant for almost the whole frequency range $(f>200 \mathrm{~Hz})$. Therefore, the annular ABH reveals as a very effective way to suppress noise radiation from cylindrical shells although its effectiveness may obviously vary from point to point (see the differences in pressure patterns at $r=10 \mathrm{~m}$ in the previous Fig. 8). In this sense, it is worth mentioning that only point excitation at a particular point has been considered and further exploration with other points at type of excitations will be worth exploring in the future.

\section{Conclusions}

In this paper, we have suggested a semi-analytical approach to characterize sound radiation by cylindrical shells with embedded annular acoustic back holes (ABHs). The case of an external point excitation on the $\mathrm{ABH}$ cylinder has been addressed. The shell velocity distribution has been computed using the Gaussian expansion method and the acoustic pressure has been obtained from the direct solution of the Helmholtz equation using Green's function in cylindrical coordinates. The surface acoustic pressure has been derived by dividing the cylindrical shell into small radiators and making use of an impedance matrix to relate the pressure to the surface radial velocity. To locate sound sources in the ABH cylinder surface, a procedure has been proposed to compute the supersonic intensity (SSI) in the spatial domain. The strategy has been validated against finite element method (FEM) simulations and has served to compute the ABH cylinder sound power level, radiation efficiency, sound intensity, supersonic sound intensity and far-field acoustic pressure. The latter have been compared for an ABH cylinder and a cylinder with uniform thickness.

The main outcomes from the performed simulations are as follows. A thick acoustic shell has been considered so that the critical frequency is smaller than the ring one. It has been demonstrated that the 
annular $\mathrm{ABH}$ has a profound impact on the cylinder mean squared velocity (MSV). For a uniform cylinder, the MSV increases up to the critical frequency, $f_{c}$, then remains more or less constant in average up to the ring frequency, $f_{R}$, and finally rapidly decreases once surpassed $f_{R}$. The sound power level (SWL) exhibits identical tendency. The MSV and SWL of the annular ABH cylinder follow the same behaviour of the uniform one but levels are reduced in about 10-15 dB beyond the critical frequency. However, the trends for the radiation efficiency, $\sigma$, are very different. While for the uniform shell $\sigma$ augments with frequency reaching a maximum close to 2 at $f_{c}$, then decreases until $f_{R}$ and then remains nearly constant past the ring frequency, the situation is neatly distinct for the $\mathrm{ABH}$ cylinder. The radiation efficiency for the latter also increases with frequency but barely surpasses a value of 1 at $f_{c}$, then remains almost constant up to $f_{R}$ and finally diminishes after it. The radiation efficiency for the ABH shell is clearly smaller than for uniform one, which inhibits sound radiation. In what concerns the source location on the shell surface, supersonic intensity (SSI) and acoustic intensity (SI) show very similar patterns for the uniform shell but clearly differ for the $\mathrm{ABH}$. It is observed that once surpassed $f_{c}$, the $\mathrm{ABH}$ becomes very efficient and sound sources are located in the close vicinity of the excitation point. The supersonic flexural waves entering the ABH become subsonic once past a given axial position and their radiation efficiency drastically diminishes. All in all proves that embedding an annular $\mathrm{ABH}$ on a cylindrical shell results in a clear reduction of the far-field radiated sound.

To finish this paper, we would like to remark that the current work only constitutes a first step showing the potential of ABHs to reduce noise radiated from cylindrical shells. In future works it will be worth exploring the influence of different types of excitations like distributed mechanical forces, incident acoustic fields or turbulent boundary layers. Also, it will be useful to check the influence of various types of boundary conditions as well as the inclusion of ring and axial stiffeners to keep the structural rigidity of the shell to acceptable values.

\section{Acknowledgements}

This work was carried out while the first author was performing a two-year PhD stay at La Salle, Universitat Ramon Llull, funded by the National Natural Science Foundation of China under Grant (51875061) and the China Scholarship Council (CSC No.201806050075). Part of it was completed during a visiting period of the first author at INSA Lyon within the framework of the LABEX CeLyA (ANR-10-LABX-0060) of Universit de Lyon, within the program Investissements d'Avenir (ANR-16-IDEX-0005) operated by the French National Research Agency (ANR). The authors gratefully acknowledge these supports as well as the in-kind assistance from La Salle, Universitat Ramon Llull, INSA Lyon and the Chongqing University to make that collaboration possible.

\section{References}

[1] A. Pelat, F. Gautier, S. C. Conlon, F. Semperlotti, The acoustic black hole: A review of theory and applications, J. Sound Vib. (2020) 115316.

[2] S. Conlon, J. Fahnline, F. Semperlotti, Numerical analysis of the vibroacoustic properties of plates with embedded grids of acoustic black holes, J. Acoust. Soc. Am. 137 (2015) 447-457.

[3] P. A. Feurtado, S. C. Conlon, Wavenumber transform analysis for acoustic black hole design, J. Acoust. Soc. Am. 140 (2016) $718-727$

[4] L. Ma, L. Cheng, Sound radiation and transonic boundaries of a plate with an acoustic black hole, J. Acoust. Soc. Am. 145 (2019) 164-172.

[5] E. G. Williams, Supersonic acoustic intensity, J. Acoust. Soc. Am. 97 (1995) 121-127.

[6] E. G. Williams, Supersonic acoustic intensity on planar sources, J. Acoust. Soc. Am. 104 (1998) $2845-2850$.

[7] L. Ma, L. Cheng, Topological optimization of damping layout for minimized sound radiation of an acoustic black hole plate, J. Sound Vib. 458 (2019) 349-364.

[8] L. Tang, L. Cheng, Impaired sound radiation in plates with periodic tunneled acoustic black holes, Mech. Syst. Signal Pr. 135 (2020) 106410.

[9] H. Ji, X. Wang, J. Qiu, L. Cheng, Y. Wu, C. Zhang, Noise reduction inside a cavity coupled to a flexible plate with embedded 2-d acoustic black holes, J. Sound Vib. 455 (2019) 324-338.

[10] P. A. Feurtado, S. C. Conlon, Transmission loss of plates with embedded acoustic black holes, J. Acoust. Soc. Am. 142 (2017) 1390-1398. 
[11] X. Du, D. Huang, Q. Fu, J. Zhang, Effects of acoustic black hole parameters and damping layer on sound insulation performance of abh circular plate, Appl. Sci. 9 (2019) 5366.

[12] J. Deng, O. Guasch, L. Maxit, L. Zheng, Transmission loss of plates with multiple embedded acoustic black holes using statistical modal energy distribution analysis, Mech. Syst. Signal Pr. 150 (2021) 107262.

[13] E. Bowyer, V. V. Krylov, Experimental study of sound radiation by plates containing circular indentations of power-law profile, Appl. Acoust. 88 (2015) 30-37.

[14] P. A. Feurtado, S. C. Conlon, An experimental investigation of acoustic black hole dynamics at low, mid, and high frequencies, J. Vib. Acoust. 138 (2016).

[15] L. Ma, L. Cheng, Numerical and experimental benchmark solutions on vibration and sound radiation of an acoustic black hole plate, Appl. Acoust. 163 (2020) 107223.

[16] J. Y. Lee, W. Jeon, Vibration damping using a spiral acoustic black hole, J. Acoust. Soc. Am. 141 (2017) 1437-1445.

[17] S. Park, M. Kim, W. Jeon, Experimental validation of vibration damping using an archimedean spiral acoustic black hole, J. Sound Vib. 459 (2019) 114838.

[18] V. V. Krylov, Propagation of plate bending waves in the vicinity of one-and two-dimensional acoustic black holes, in: ECCOMAS Thematic Conference on Computational Methods in Structural Dynamics and Earthquake Engineering, Rethymno, Crete (Greece) 13-16 June, 2007.

[19] V. Georgiev, J. Cuenca, F. Gautier, L. Simon, V. Krylov, Damping of structural vibrations in beams and elliptical plates using the acoustic black hole effect, J. Sound Vib. 330 (2011) 2497-2508.

[20] O. Aklouche, A. Pelat, S. Maugeais, F. Gautier, Scattering of flexural waves by a pit of quadratic profile inserted in an infinite thin plate, J. Sound Vib. 375 (2016) 38-52.

[21] D. O'Boy, V. Krylov, Damping of flexural vibrations in circular plates with tapered central holes, J. Sound Vib. 330 (2011) 2220-2236.

[22] D. J. OBoy, E. P. Bowyer, V. V. Krylov, Point mobility of a cylindrical plate incorporating a tapered hole of power-law profile, J. Acoust. Soc. Am. 129 (2011) 3475-3482.

[23] D. O'Boy, V. Krylov, Vibration of a rectangular plate with a central power-law profiled groove by the rayleigh-ritz method, Appl. Acoust. 104 (2016) 24-32.

[24] T. Zhou, L. Tang, H. Ji, J. Qiu, L. Cheng, Dynamic and static properties of double-layered compound acoustic black hole structures, Int. J. Appl. Mech. 9 (2017) 1750074.

[25] J. Deng, O. Guasch, L. Zheng, Ring-shaped acoustic black holes for broadband vibration isolation in plates, J. Sound Vib. 458 (2019) 109-122.

[26] J. Deng, O. Guasch, L. Zheng, A semi-analytical method for characterizing vibrations in circular beams with embedded acoustic black holes, J. Sound Vib. (2020) 115307.

[27] L. Tang, L. Cheng, Periodic plates with tunneled acoustic-black-holes for directional band gap generation, Mech. Syst. Signal Pr. 133 (2019) 106257.

[28] J. Deng, O. Guasch, L. Maxit, L. Zheng, Annular acoustic black holes to reduce propagative Bloch-Floquet flexural waves in periodically supported cylindrical shells, in: INTER-NOISE and NOISE-CON Congress and Conference Proceedings, volume 259 (9), Institute of Noise Control Engineering, pp. 206-212, 2019.

[29] J. Deng, O. Guasch, L. Maxit, L. Zheng, Reduction of Bloch-Floquet bending waves via annular acoustic black holes in periodically supported cylindrical shell structures, Appl. Acoust. 169 (2020) 107424.

[30] J. Deng, O. Guasch, L. Maxit, L. Zheng, Vibration of cylindrical shells with embedded annular acoustic black holes using the Rayleigh-Ritz method with Gaussian basis functions, Mech. Syst. Signal Pr. 150 (2021) 107225.

[31] F. J. Fahy, P. Gardonio, Sound and structural vibration: radiation, transmission and response, Elsevier, 2007.

[32] C. Wang, J. Lai, The sound radiation efficiency of finite length acoustically thick circular cylindrical shells under mechanical excitation i: theoretical analysis, J. Sound Vib. 232 (2000) 431-447.

[33] E. G. Williams, Fourier acoustics: sound radiation and nearfield acoustical holography, Elsevier, 1999.

[34] E. Szechenyi, Modal densities and radiation efficiencies of unstiffened cylinders using statistical methods, J. Sound Vib. 19 (1971) 65-81.

[35] L. Maxit, M. Karimi, V. Meyer, N. Kessissoglou, Vibroacoustic responses of a heavy fluid loaded cylindrical shell excited by a turbulent boundary layer, J. Fluid Struct. 92 (2020) 102758.

[36] L. Maxit, O. Guasch, V. Meyer, M. Karimi, Noise radiated from a periodically stiffened cylindrical shell excited by a turbulent boundary layer, J. Sound Vib. 466 (2020) 115016.

[37] J. Deng, L. Zheng, P. Zeng, Y. Zuo, O. Guasch, Passive constrained viscoelastic layers to improve the efficiency of truncated acoustic black holes in beams, Mech. Syst. Signal Pr. 118 (2019) 461-476.

[38] J. Deng, L. Zheng, O. Guasch, H. Wu, P. Zeng, Y. Zuo, Gaussian expansion for the vibration analysis of plates with multiple acoustic black holes indentations, Mech. Syst. Signal Pr. 131 (2019) 317-334.

[39] L. Tang, L. Cheng, H. Ji, J. Qiu, Characterization of acoustic black hole effect using a one-dimensional fully-coupled and wavelet-decomposed semi-analytical model, J. Sound Vib. 374 (2016) 172-184.

[40] L. Tang, L. Cheng, Broadband locally resonant band gaps in periodic beam structures with embedded acoustic black holes, J. Appl. Phys. 121 (2017) 194901

[41] L. Ma, S. Zhang, L. Cheng, A 2D Daubechies wavelet model on the vibration of rectangular plates containing strip indentations with a parabolic thickness profile, J. Sound Vib. 429 (2018) 130-146.

[42] P. R. Stepanishen, Radiated power and radiation loading of cylindrical surfaces with nonuniform velocity distributions, J. Acoust. Soc. Am. 63 (1978) 328-338.

[43] Y. Sun, T. Yang, Y. Chen, Sound radiation modes of cylindrical surfaces and their application to vibro-acoustics analysis of cylindrical shells, J. Sound Vib. 424 (2018) 64-77. 
[44] M. B. S. Magalhães, R. A. Tenenbaum, Supersonic acoustic intensity for arbitrarily shaped sources, Acta Acust. united Ac. 92 (2006) 189-201.

[45] E. Fernandez-Grande, F. Jacobsen, Q. Leclere, Direct formulation of the supersonic acoustic intensity in space domain, J. Acoust. Soc. Am. 131 (2012) 186-193.

[46] S. Marburg, E. Lösche, H. Peters, N. Kessissoglou, Surface contributions to radiated sound power, J. Acoust. Soc. Am. 133 (2013) 3700-3705.

[47] E. G. Williams, Convolution formulations for non-negative intensity, J. Acoust. Soc. Am. 134 (2013) 1055-1066.

[48] D. Liu, H. Peters, S. Marburg, N. Kessissoglou, Supersonic intensity and non-negative intensity for prediction of radiated sound, J. Acoust. Soc. Am. 139 (2016) 2797-2806.

[49] B. Sivasubramonian, G. Rao, A. Krishnan, Free vibration of longitudinally stiffened curved panels with cutout, J. Sound Vib. 226 (1999) 41-55. 